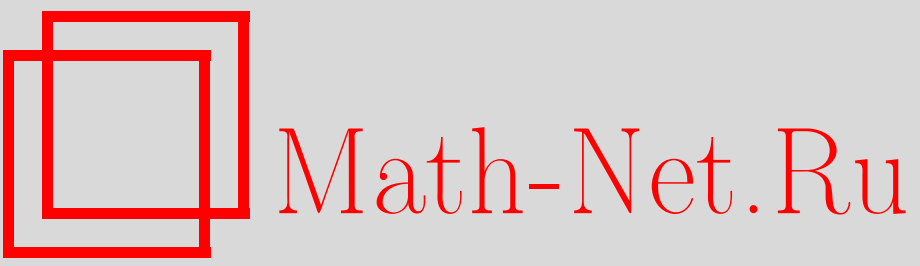

Д. Б. Рохлин, Рекуррентные формулы для границ цен платежных обязательств в моделях рынков с дискретным временем, Теория вероятн. и ее примен., 2011, том 56, выпуск $1,47-76$

DOI: https://doi.org/10.4213/tvp4323

Использование Общероссийского математического портала Math-Net.Ru подразумевает, что вы прочитали и согласны с пользовательским соглашением

http: //www . mathnet.ru/rus/agreement

Параметры загрузки:

IP: 54.162 .127 .20

26 апреля 2023 г., 08:19:10 


\title{
РЕКУРРЕНТНЫЕ ФОРМУЛЫ ДЛЯ ГРАНИЦ ЦЕН ПЛАТЕЖНЫХ ОБЯЗАТЕЛЬСТВ В МОДЕЛЯХ РЫНКОВ С ДИСКРЕТНЫМ ВРЕМЕНЕМ
}

\begin{abstract}
Вычисление границ цен платежных обязательств сведено к решению последовательности связанных между собой конечномерных оптимизационных задач, зависящих от параметра. В модели идеального рынка полученные формулы описывают верхнюю цену хеджирования, а в модели мультивалютного рынка с операционными издержками - множество начальных портфелей, позволяющих суперхеджировать векторное платежное обязательство. Указанные формулы не содержат мартингальных мер и их аналогов. Доказательства основаны на теореме о мартингальном выборе. Эффективность предлагаемого подхода иллюстрируется рядом примеров.
\end{abstract}

Ключевые слова и фразы: цены хеджирования, операционные издержки, дискретное время, теорема о мартингальном выборе, измеримые многозначные отображения, выпуклость.

1. Основные результаты. Определение границ множества справедливых цен платежных обязательств является одной из основных задач финансовой математики. В данной работе рассматриваются модели рынков на общих вероятностных пространствах в случае конечного дискретного времени. При таких предположениях указанная задача хорошо изучена как для классической модели идеального рынка, так и для моделей рынков с операционными издержками.

Пусть имеется вероятностное пространство $(\Omega, \mathscr{F}, \mathbf{P})$, наделенное фильтрацией $\mathbf{F}=\left(\mathscr{F}_{t}\right)_{t=0}^{T}$, где $\mathscr{F}_{0}=\{\varnothing, \Omega\}, \mathscr{F}_{T}=\mathscr{F}$. Будем предполагать, что $\sigma$-алгебры $\mathscr{F}_{t}$ полны относительно меры $\mathbf{P}$. Модель идеального рынка определяется заданием согласованного с фильтрацией $d$-мерного процесса $\widehat{S}_{t}=\left(\widehat{S}_{t}^{1}, \ldots, \widehat{S}_{t}^{d}\right), d \geqslant 2$, цен рисковых активов. Предположим, что $\widehat{S}_{t}^{i}>0$, и произведем дисконтирование, т.е. выразим цены всех активов в единицах первого: $S_{t}^{i}=\widehat{S}_{t}^{i} / \widehat{S}_{t}^{1}$. Дисконтированный капитал $X_{t}^{\gamma}$

*Южный федеральный университет, факультет математики, механики и компьютерных наук, ул. Мильчакова, 8А, 344090 Ростов-на-Дону, Россия; е-таil: rokhlin@math.rsu.ru 
инвестора представляет собой сумму неслучайного начального капитала $x$ и выигрыша $G_{t}^{\gamma}$ при ставках на приращения цен:

$$
X_{t}^{\gamma}=x+G_{t}^{\gamma}=x+\sum_{k=1}^{t} \gamma_{k} \cdot \Delta S_{k}, \quad \Delta S_{k}=S_{k}-S_{k-1}, \quad 1 \leqslant t \leqslant T .
$$

Здесь $\left(\gamma_{t}^{2}, \ldots, \gamma_{t}^{d}\right)$ - предсказуемый процесс портфеля, а $a \cdot b$ обозначает скалярное произведение. Будем считать, что модель удовлетворяет условию отсутствия арбитража, т.е. из неравенства $G_{T}^{\gamma} \geqslant 0$ п.н. (по мере P) вытекает, что $G_{T}^{\gamma}=0$ п.н.

Пусть $L^{0}(\mathscr{F})$ - множество классов эквивалентности $\mathscr{F}$-измеримых вещественнозначных случайных величин. Обозначим $\mathbf{C}^{*}\left(f_{T}\right)$ минимальный начальный капитал, позволяющий продавцу хеджировать платежное обязательство, представленное случайной величиной $f_{T} \in L^{0}\left(\mathscr{F}_{T}\right)$ :

$\mathbf{C}^{*}\left(f_{T}\right)=\inf \left\{x: x+G_{T}^{\gamma} \geqslant f_{T}\right.$ для некоторого предсказуемого процесса $\left.\gamma\right\}$.

Данная величина называется верхней ценой хеджирования (или ченой продавча). Аналогичным образом определяется нижняя чена хеджирования (чена покупателя):

$\mathbf{C}_{*}\left(f_{T}\right)=\sup \left\{x: f_{T}+G_{T}^{\gamma} \geqslant x\right.$ для некоторого предсказуемого процесса $\left.\gamma\right\}$.

Величина $\mathbf{C}_{*}\left(f_{T}\right)$ представляет собой наибольшую сумму денег, которую без риска можно заплатить при покупке $f_{T}$.

Введем множество $\mathscr{P}(S)$ эквивалентных $\mathbf{P}$ вероятностных мер $\mathbf{Q}$ (обозначение $\mathbf{Q} \sim \mathbf{P}$ ), относительно которых процесс $S$ является мартингалом. Хорошо известно, что имеют место равенства

$$
\begin{aligned}
& \mathbf{C}_{*}\left(f_{T}\right)=\inf \left\{\mathbf{E}_{\mathbf{Q}} f_{T}: \mathbf{Q} \in \mathscr{P}(S), \mathbf{E}_{\mathbf{Q}}\left|f_{T}\right|<\infty\right\}, \\
& \mathbf{C}^{*}\left(f_{T}\right)=\sup \left\{\mathbf{E}_{\mathbf{Q}} f_{T}: \mathbf{Q} \in \mathscr{P}(S), \mathbf{E}_{\mathbf{Q}}\left|f_{T}\right|<\infty\right\} .
\end{aligned}
$$

Для ограниченных снизу случайных величин $f_{T}$ доказательство равенства (1.1) можно найти в [8] (теорема 2.1.11), а для ограниченных $f_{T}$ в [25] (гл. $6, \S 1 \mathrm{c}$, теорема 1). В общем случае данные утверждения вытекают, например, из результатов работы [21] (теорема 4.1). Множество «справедливых» цен $f_{T}$ заключено между $\mathbf{C}_{*}\left(f_{T}\right)$ и $\mathbf{C}^{*}\left(f_{T}\right)$ (см. $[2$, гл. 2], [8, гл. 2]).

Несмотря на теоретическую важность указанного двойственного описания цен продавца и покупателя, непосредственное применение формул (1.1), (1.2) может оказаться затруднительным, так как обычно множество мартингальных мер является очень большим. В представленные ниже рекуррентные формулы мартингальные меры не входят, а вычисления сводятся к решению последовательности связанных между собой конечномерных оптимизационных задач, зависящих от параметра. 
Для формулировки результатов работы нам потребуются определения измеримого интегранда $[14$, гл. $14 \mathrm{D}]$ и регулярного условного математического ожидания [3]. Расширенная функция $\psi: \Omega \times \mathbf{R}^{d} \rightarrow[-\infty,+\infty]$ называется $\mathscr{F}$-измеримым интеграндом, если функция $\psi(\cdot, x)$ является $\mathscr{F}$-измеримой для любого $x \in \mathbf{R}^{d}$.

Многозначное отображение $G$, ставящее в соответствие каждому $\omega \in \Omega$ некоторое множество $G(\omega) \subset \mathbf{R}^{d}$, называется $\mathscr{F}$-измеримым, если $\{\omega: G(\omega) \cap V \neq \varnothing\} \in \mathscr{F}$ для любого открытого множества $V \subset \mathbf{R}^{d}$. Интегранд называется нормальныл, если его надграфик

$$
\omega \mapsto \operatorname{epi} \psi(\omega, \cdot)=\{(x, y): f(\omega, x) \leqslant y\} \subset \mathbf{R}^{d} \times \mathbf{R}
$$

является замкнутым и $\mathscr{F}$-измеримым. Используя терминологию стохастического анализа, назовем интегранды $\psi_{1}, \psi_{2}$ неразличимыми, если множество $A=\left\{(\omega, x): \psi_{1}(\omega, x) \neq \psi_{2}(\omega, x)\right\}$ является пренебрежимыл, т.е. $\mathbf{P}\left\{\omega:\left(\omega \times \mathbf{R}^{d}\right) \cap A \neq \varnothing\right\}=0$. Фактически, мы будем рассматривать классы эквивалентности неразличимых интеграндов. Через $L^{0}(\mathscr{F}, G)$ обозначим множество классов эквивалентности $\mathscr{F}$-измеримых случайных векторов, принимающих значения в $G$ п.н.

Рассмотрим случайную величину $f(\omega, z)$, зависящую от параметра $z \in \mathbf{R}^{k}$. Пусть $f$ ограничена и $\mathscr{F} \otimes \mathscr{B}\left(\mathbf{R}^{k}\right)$-измерима, где $\mathscr{B}\left(\mathbf{R}^{k}\right)-$ борелевская $\sigma$-алгебра пространства $\mathbf{R}^{k}$. Положим $f_{\varphi}(\omega)=f(\omega, \varphi(\omega))$. Функция

$$
\widehat{f}=\mathbf{E}^{r}(f \mid \mathscr{H}): \Omega \times \mathbf{R}^{d} \rightarrow \mathbf{R}
$$

называется регулярным условным математическим ожиданием (см. [3]) случайной величины $f$ относительно $\sigma$-алгебры $\mathscr{H} \subset \mathscr{F}$, если

(i) $\widehat{f}$ является $\mathscr{H} \otimes \mathscr{B}\left(\mathbf{R}^{d}\right)$-измеримой,

(ii) $\widehat{f}_{\varphi}=\mathbf{E}\left(f_{\varphi} \mid \mathscr{H}\right)$ п.н. для любой $\mathscr{H}$-измеримой функции $\varphi: \Omega \rightarrow \mathbf{R}^{d}$.

В работе [3] установлено, что регулярное условное математическое ожидание существует и единственно с точностью до неразличимости.

Полезно рассмотреть частный случай. Пусть $W \in L^{0}\left(\mathscr{F}, \mathscr{B}\left(\mathbf{R}^{m}\right)\right)$ и $f(\omega, z)=g(\omega, W(\omega), z)$, где $g$ есть $\mathscr{H} \otimes \mathscr{B}\left(\mathbf{R}^{m}\right) \otimes \mathscr{B}\left(\mathbf{R}^{k}\right)$-измеримая ограниченная функция. Обозначим $\mu(\omega, d x)$ регулярное условное распределение $W$ относительно $\mathscr{H}$. Легко видеть, что

$$
\mathbf{E}^{r}(g(\omega, W, z) \mid \mathscr{H})=\int_{\mathbf{R}^{m}} g(\omega, x, z) \mu(\omega, d x) \quad \text { п.н. }
$$

Правая часть данного соотношения определяет одну из версий регулярного условного математического ожидания.

Пусть $\mathbf{I}(A)(x)=1$, если $x \in A$, и $\mathbf{I}(A)(x)=0$, если $x \notin A$. 
Теорема 1. Определим согласованнье с фильтрачией $\mathbf{F}$ последовательности $\left(w_{t}^{u}\right)_{t=0}^{T},\left(w_{t}^{l}\right)_{t=0}^{T}$ посредством рекуррентных формул:

$$
\begin{aligned}
w_{T}^{u} & =w_{T}^{l}=f_{T}, \\
w_{t}^{u}(\omega) & =\inf \left\{\alpha+\beta \cdot S_{t}(\omega):(\alpha, \beta) \in G_{t}^{u}(\omega)\right\}, \\
w_{t}^{l}(\omega) & =\sup \left\{\alpha+\beta \cdot S_{t}(\omega):(\alpha, \beta) \in G_{t}^{l}(\omega)\right\}, \\
G_{t}^{u} & =\left\{(\alpha, \beta) \in \mathbf{R} \times \mathbf{R}^{d-1}: \mathbf{E}^{r}\left(\mathbf{I}\left(\alpha+\beta \cdot S_{t+1} \geqslant w_{t+1}^{u}\right) \mid \mathscr{F}_{t}\right)(\omega, \alpha, \beta)=1\right\}, \\
G_{t}^{l} & =\left\{(\alpha, \beta) \in \mathbf{R} \times \mathbf{R}^{d-1}: \mathbf{E}^{r}\left(\mathbf{I}\left(\alpha+\beta \cdot S_{t+1} \leqslant w_{t+1}^{l}\right) \mid \mathscr{F}_{t}\right)(\omega, \alpha, \beta)=1\right\} .
\end{aligned}
$$

(i) Если $G_{m}^{u}=\varnothing$ (соответственно $\left.G_{m}^{l}=\varnothing\right)$ на множестве положительной меры для некоторого $m$, mо $\mathbf{C}^{*}\left(f_{T}\right)=+\infty$ (соответственно $\left.\mathbf{C}_{*}\left(f_{T}\right)=-\infty\right)$.

(ii) В противном случае $\mathbf{C}^{*}\left(f_{T}\right)=w_{0}^{u}, \mathbf{C}_{*}\left(f_{T}\right)=w_{0}^{l}$.

Формулы теоремы 1 напоминают рекуррентные соотношения метода динамического программирования. Близкие результаты получены в работе [1]. В отличие от [1] мы не используем операцию существенного супремума семейства случайных величин: оптимизационные задачи, определяющие функции $w_{t}^{u}, w_{t}^{l}$, конечномерны. Кроме того, мы не предполагаем, что фильтрация $\mathbf{F}$ порождается процессом $S$.

Заметим, что достаточно было ограничиться рассмотрением только одного набора рекуррентных соотношений, например, для верхней цены хеджирования. Действительно, $\mathbf{C}_{*}\left(f_{T}\right)=-\mathbf{C}^{*}\left(-f_{T}\right)$ и аналогичными равенствами связаны функции $w_{t}^{l}$ и $w_{t}^{u}$. Формула для верхней цены хеджирования чаще используется в приложениях. С другой стороны, как будет видно в дальнейшем, формулы для нижней цены имеют более естественный вид с точки зрения выпуклого анализа. Поэтому мы предпочитаем сохранить оба набора формул.

Проверим, что последовательность $\left(w_{t}^{l}\right)$ корректно определена. Если функция $w_{t+1}^{l}$ является $\mathscr{F}_{t+1}$-измеримой, то функция

$$
f(\omega, \alpha, \beta)=\mathbf{I}\left(\alpha+\beta \cdot S_{t+1}(\omega) \geqslant w_{t+1}^{u}(\omega)\right)
$$

измерима относительно $\mathscr{F}_{t+1} \otimes \mathscr{B}(\mathbf{R}) \otimes \mathscr{B}\left(\mathbf{R}^{d-1}\right)$ и полунепрерывна сверху по $(\alpha, \beta)$ при фиксированном $\omega$. Согласно [3, теорема 2.2], функция $\widehat{f}=\mathbf{E}^{r}\left(f \mid \mathscr{F}_{t}\right)(\omega, \alpha, \beta)$ также полунепрерывна сверху по $(\alpha, \beta)$ при почти всех $\omega$. Кроме того, по определению, эта функция является $\mathscr{F}_{t} \otimes \mathscr{B}(\mathbf{R}) \otimes \mathscr{B}\left(\mathbf{R}^{d-1}\right)$-измеримой. Отсюда следует, что $(-\widehat{f})-$ нормальный интегранд [14, следствие 14.34]. Но тогда многозначное отображение

$$
\omega \mapsto G_{t}^{l}(\omega)=\{(\alpha, \beta): \widehat{f}(\omega, \alpha, \beta)=1\}=\{(\alpha, \beta): \widehat{f}(\omega, \alpha, \beta) \geqslant 1\}
$$


является $\mathscr{F}_{t}$-измеримым [14, предложение 14.33]. Здесь учтено, что $\widehat{f} \leqslant 1$ в силу неравенства $f \leqslant 1$. Пример 14.51 из [14] показывает теперь, что $w_{t}^{l}$ измерима относительно $\mathscr{F}_{t}$.

Далее основное внимание будет уделено более общей модели рынка с операционными издержками (или рынка с «трением»). Имеется ряд альтернативных моделей такого рода: см. [8, гл. 3]. Мы рассматриваем предложенную в работе [21] модель «рынка обмена» (barter market), которая наилучшим образом укладывается в схему рассуждений настоящей работы. Для наглядности активы будем считать валютами.

Итак, пусть имеется согласованный с фильтрацией процесс $(d \times d)$ матриц обменных курсов $\Pi_{t}=\left(\pi_{t}^{i j}\right)_{i, j=1}^{d}$. Элемент $\pi_{t}^{i j}$ определяет количество единиц $i$-й валюты, которое можно обменять на единицу $j$-й валюты в момент времени $t$. Предполагается, что выполняются соотношения $\pi^{i j}>0, \pi^{i i}=1$ и $\pi^{i j} \leqslant \pi^{i k} \pi^{k j}$, последнее из которых означает, что прямой обмен не хуже, чем цепочка обменов. Конусом платежеспособности называется выпуклый конус $K_{t}$, порожденный векторами стандартного базиса $\left\{e_{i}\right\}_{i=1}^{d}$ и векторами $\pi_{t}^{i j} e_{i}-e_{j}$.

Пусть компоненты $\mathscr{F}_{t}$-измеримого случайного вектора $\theta_{t}=$ $\left(\theta_{t}^{1}, \ldots, \theta_{t}^{d}\right)$ описывают количество единиц каждой валюты в портфеле инвестора в момент времени $t$. Элементами $K_{t}$ являются портфели, которые могут быть ликвидированы. Процесс портфеля $\left(\theta_{t}\right)_{t=0}^{d}$ называется самофинансируемьм, если его приращения могут быть «приобретены бесплатно»:

$$
\theta_{t}-\theta_{t-1} \in-K_{t} \quad \text { п.н., } \quad t=0, \ldots, T ; \quad \theta_{-1}=0 .
$$

Обозначим $A_{T}(\Pi)$ выпуклый конус в множестве $L^{0}\left(\mathscr{F}_{T}, \mathbf{R}^{d}\right)$, состоящий из элементов $\theta_{T}$ самофинансируемых портфелей. Говорят (см. [21]), что процесс обменных курсов $\left(\Pi_{t}\right)_{t=0}^{T}$ удовлетворяет условию робастного отсутствия арбитража ( $\mathrm{NA}^{r}$ : robust no-arbitrage), если существует процесс $\left(\widetilde{\Pi}_{t}\right)_{t=0}^{T}$ обменных курсов с меньшими операционными издержками:

$$
\left[\frac{1}{\widetilde{\pi}_{t}^{j i}}, \widetilde{\pi}_{t}^{i j}\right] \subset \mathrm{ri}\left[\frac{1}{\pi_{t}^{j i}}, \pi_{t}^{i j}\right],
$$

удовлетворяющий условию отсутствия арбитража (NA):

$$
A_{T}(\widetilde{\Pi}) \cap L^{0}\left(\mathscr{F}_{T}, \mathbf{R}_{+}^{d}\right)=\{0\}, \quad \mathbf{R}_{+}^{d}=\left\{x \in \mathbf{R}^{d}: x^{i} \geqslant 0\right\} .
$$

Здесь и далее ri обозначает относительную внутренность множества (см. [13, гл. $2, \S 6])$.

Пусть $\mathbf{H}_{+}=\mathbf{H}_{+}\left(\zeta_{T}\right)$ обозначает множество портфелей $\zeta_{0} \in \mathbf{R}^{d}$, которые позволяют суперхеджировать платежное векторное обязательство $\zeta_{T} \in L^{0}\left(\mathscr{F}_{T}, \mathbf{R}^{d}\right)$ :

$$
\mathbf{H}_{+}\left(\zeta_{T}\right)=\left\{\zeta_{0} \in \mathbf{R}^{d}: \zeta_{T}-\zeta_{0} \in A_{T}(\Pi)\right\} .
$$


Аналогичным образом определяется множество $\mathbf{H}_{-}$портфелей, позволяющих субхеджировать $\zeta_{T}: \mathbf{H}_{-}\left(\zeta_{T}\right)=\left\{\zeta_{0} \in \mathbf{R}^{d}: \zeta_{0}-\zeta_{T} \in A_{T}(\Pi)\right\}$.

Пусть $\mathscr{M}\left(\right.$ ri $\left.K^{*}\right)$ обозначает множество $\mathbf{P}$-мартингалов, элементы $Z_{t}$ которых принимают значения в относительной внутренности конусов $K_{t}^{*}=\left\{y: x \cdot y \geqslant 0, x \in K_{t}\right\}$, сопряженных к $K_{t}: Z_{t} \in \operatorname{ri} K_{t}^{*}$. В работе [21] элементы $\mathscr{M}\left(\right.$ ri $\left.K^{*}\right)$ называются строго согласованными процессами цен. При выполнении условия $\mathrm{NA}^{r}$ множество $\mathscr{M}\left(\mathrm{ri} K^{*}\right)$ непусто [21, теорема 1.7], а множество $\mathbf{H}_{+}$допускает двойственное описание (см. [21, теорема 4.1]):

$$
\mathbf{H}_{+}\left(\zeta_{T}\right)=\left\{\zeta_{0} \in \mathbf{R}^{d}: \zeta_{0} \cdot Z_{0} \geqslant \mathbf{E}\left(\zeta_{T} \cdot Z_{T}\right), Z \in \mathscr{M}\left(\operatorname{ri} K^{*}, \zeta_{T}\right)\right\} .
$$

Здесь $\mathscr{M}\left(\right.$ ri $\left.K^{*}, \zeta_{T}\right)$ - множество элементов $\mathscr{M}\left(\right.$ ri $\left.K^{*}\right)$, удовлетворяющих дополнительному условию $\mathbf{E}\left|\zeta_{T} \cdot Z_{T}\right|<\infty$. Аналогичным образом описывается и $\mathbf{H}_{-}$:

$$
\mathbf{H}_{-}\left(\zeta_{T}\right)=\left\{\zeta_{0} \in \mathbf{R}^{d}: \zeta_{0} \cdot Z_{0} \leqslant \mathbf{E}\left(\zeta_{T} \cdot Z_{T}\right), Z \in \mathscr{M}\left(\operatorname{ri~} K^{*}, \zeta_{T}\right)\right\} .
$$

Нетрудно проверить (см. [21]), что

$$
\operatorname{ri} K_{t}^{*}=\left\{w \in \mathbf{R}_{++}^{d}: \frac{w^{j}}{w^{i}} \in \operatorname{ri}\left[\frac{1}{\pi_{t}^{j i}}, \pi_{t}^{i j}\right], 1 \leqslant i, j \leqslant d\right\}
$$

где $\mathbf{R}_{++}^{d}-$ множество векторов со строго положительными компонентами.

Введем опорную и индикаторную функции множества $B \subset \mathbf{R}^{d}$ :

$$
s(x \mid B)=\sup _{y \in B}(x \cdot y), \quad \delta(x \mid B)= \begin{cases}0, & x \in B, \\ +\infty, & x \notin B,\end{cases}
$$

а также субдифференциал сублинейной функции $p$ в нуле:

$$
\partial p=\left\{x: x \cdot y \leqslant p(y), y \in \mathbf{R}^{d}\right\} .
$$

Основной результат настоящей работы содержится в теореме 2. В ней представлены рекуррентные формулы для множеств $\mathbf{H}_{+}, \mathbf{H}_{-}$.

Теорема 2. Пусть выполнено условие робастного отсутствия арбитража $\left(\mathrm{NA}^{r}\right)$. Определим согласованные с фильтрачией последовательности нормальных интеграндов $\left(h_{t}^{l}\right)_{t=0}^{T},\left(h_{t}^{u}\right)_{t=0}^{T}$ посредством рекуррентных соотношений:

$$
\begin{aligned}
h_{T}^{l}\left(\omega, x^{*}\right) & =\zeta_{T} \cdot x^{*}+\delta\left(x^{*} \mid K_{T}^{*}\right), \quad h_{T}^{u}\left(\omega, x^{*}\right)=\zeta_{T} \cdot x^{*}-\delta\left(x^{*} \mid K_{T}^{*}\right), \quad(1.5) \\
h_{t}^{l}\left(\omega, x^{*}\right) & =s\left(x^{*} \mid G_{t}^{l}\right)+\delta\left(x^{*} \mid K_{t}^{*}\right), \quad h_{t}^{u}\left(x^{*}\right)=-s\left(-x^{*} \mid G_{t}^{u}\right)-\delta\left(x^{*} \mid K_{t}^{*}\right), \\
G_{t}^{l}(\omega) & =\left\{z \in \mathbf{R}^{d}: \mathbf{E}^{r}\left(\mathbf{I}\left(z \in \partial h_{t+1}^{l}\right) \mid \mathscr{F}_{t}\right)(\omega, z)=1\right\}, \\
G_{t}^{u}(\omega) & =\left\{z \in \mathbf{R}^{d}: \mathbf{E}^{r}\left(\mathbf{I}\left(-z \in \partial\left(-h_{t+1}^{u}\right)\right) \mid \mathscr{F}_{t}\right)(\omega, z)=1\right\} .
\end{aligned}
$$


(i) Eсли $G_{m}^{u}=\varnothing\left(\right.$ соответственно $\left.G_{m}^{l}=\varnothing\right)$ на множестве положительной меры для некоторого $m$, то $\mathbf{H}_{+}\left(\zeta_{T}\right)=\varnothing$ (соответственно $\left.\mathbf{H}_{-}\left(\zeta_{T}\right)=\varnothing\right)$. При этом $h_{t}^{u}, h_{t}^{l}, t \leqslant m$, не определеньи.

(ii) B противном случае $\mathbf{H}_{-}\left(\zeta_{T}\right)=\partial h_{0}^{l}, \mathbf{H}_{+}\left(\zeta_{T}\right)=-\partial\left(-h_{0}^{u}\right)$.

Отметим, что имеет место равенство $\mathbf{H}_{-}\left(\zeta_{T}\right)=-\mathbf{H}_{+}\left(-\zeta_{T}\right)$, и аналогичные соотношения связывают функции $h_{t}^{l}$ и $h_{t}^{u}$. Поэтому один из указанных в теореме 2 наборов рекуррентных соотношений по существу является излишним.

Проверим, что последовательность $\left(h_{t}^{l}\right)$ корректно определена и ее элементы являются сублинейными нормальными интеграндами. Введем преобразование Юнга-Фенхеля выпуклой функции $\psi$ :

$$
\psi^{*}(x)=\sup _{y \in \mathbf{R}^{d}}(x \cdot y-\psi(y)) .
$$

Пусть $h_{t+1}-$ сублинейный нормальный $\mathscr{F}_{t+1}$-измеримый интегранд. Заметим, что

$$
f(\omega, z):=\mathbf{I}\left(z \in \partial h_{t+1}^{l}\right)=\mathbf{I}\left(h_{t+1}^{1, *}(\omega, z) \leqslant 0\right),
$$

где $h_{t+1}^{1, *}$ - преобразование Юнга-Фенхеля функции $h_{t+1}^{l}$ по второму аргументу. Но поскольку $h_{t+1}^{1, *}-$ нормальный интегранд [14, теорема $14.50]$, то функция $f$ является $\mathscr{F}_{t+1} \otimes \mathscr{B}\left(\mathbf{R}^{d}\right)$-измеримой и полунепрерывной сверху по $z$. По тем же соображениям, что и при рассмотрении последовательности $\left(w_{t}^{l}\right)$, отсюда вытекает, что $\widehat{f}=\mathbf{E}^{r}\left(f \mid \mathscr{F}_{t}\right)$ - нормальный интегранд и многозначное отображение $G_{t}^{l}$ является $\mathscr{F}_{t}$-измеримым. Но тогда $h_{t}^{l}$ является $\mathscr{F}_{t}$-измеримым нормальным интегандом (см. $[14$, пример 14.51, предложение 14.44]).

Представляет интерес конкретизация теоремы 2 для модели с избранной валютой - банковским счетом. В такой модели операция обмена актива с номером $i$ на актив с номером $j$ эквивалентна последовательному обмену $i$-го актива на банковский счет и обмену полученных денег на $j$-й актив. Если банковский счет имеет номер 1 , то элементы матрицы обменных курсов представимы в виде $\pi^{i j}=\pi^{i 1} \pi^{1 j}[5]$, [16]. При этом

$$
S^{b, i}=\frac{1}{\pi^{i, 1}}, \quad S^{a, i}=\pi^{1, i}, \quad i=2, \ldots, d,
$$

где согласованные положительные процессы $S^{b, i} \leqslant S^{a, i}$ определяют цены продажи и покупки $i$-й валюты, выраженные в единицах банковского счета. Для данной модели

$$
K_{t}^{*}=\left\{\lambda\left(1, y^{*}\right): y^{*} \in C_{t}=\left[S_{t}^{2, b}, S_{t}^{2, a}\right] \times \cdots \times\left[S_{t}^{d, b}, S_{t}^{d, a}\right], \lambda \geqslant 0\right\} .
$$

Теорема 3. Пусть модель валютного рынка с банковским счетом удовлетворяет условию $\left(\mathrm{NA}^{r}\right)$. Для заданного платежного обязатель- 
ства $\zeta_{T}=\left(\xi_{T}, \eta_{T}\right) \in L^{0}\left(\mathscr{F}_{T}, \mathbf{R} \times \mathbf{R}^{d-1}\right)$ введем согласованные с фильтраиией последовательности $\left(g_{t}^{l}\right)_{t=0}^{T},\left(g_{t}^{u}\right)_{t=0}^{T}$ по рекуррентньлм формулам

$$
\begin{aligned}
g_{T}^{l}\left(\omega, y^{*}\right) & =g_{T}^{u}\left(\omega, y^{*}\right)=\xi_{T}+\eta_{T} \cdot y^{*}, \quad y^{*} \in C_{T}, \\
g_{t}^{l}\left(\omega, y^{*}\right) & =\sup \left\{\alpha+\beta \cdot y^{*}:(\alpha, \beta) \in G_{t}^{l}\right\}, \quad y^{*} \in C_{t}, \\
g_{t}^{u}\left(\omega, y^{*}\right) & =\inf \left\{\alpha+\beta \cdot y^{*}:(\alpha, \beta) \in G_{t}^{u}\right\}, \quad y^{*} \in C_{t}, \\
G_{t}^{l}(\omega) & =\left\{(\alpha, \beta): \mathbf{E}^{r}\left(\mathbf{I}\left\{\alpha+\beta \cdot y^{*} \leqslant g_{t+1}^{l}\left(\omega, y^{*}\right), y^{*} \in C_{t+1}\right\} \mid \mathscr{F}_{t}\right)=1\right\}, \\
G_{t}^{u}(\omega) & =\left\{(\alpha, \beta): \mathbf{E}^{r}\left(\mathbf{I}\left\{\alpha+\beta \cdot y^{*} \geqslant g_{t+1}^{u}\left(\omega, y^{*}\right), y^{*} \in C_{t+1}\right\} \mid \mathscr{F}_{t}\right)=1\right\} .
\end{aligned}
$$

(i) $E c л и G_{m}^{u}=\varnothing\left(\right.$ соответственно $\left.G_{m}^{l}=\varnothing\right)$ на множестве положительной меры для некоторого $m$, то $\mathbf{H}_{+}=\varnothing$ (соответственно $\left.\mathbf{H}_{-}=\varnothing\right)$. При этом $g_{t}^{u}, g_{t}^{l}, t \leqslant m$, не определеньи.

(ii) B противном случае

$$
\begin{aligned}
& \mathbf{H}_{-}\left(\xi_{T}, \eta_{T}\right)=\left\{\left(\xi_{0}, \eta_{0}\right) \in \mathbf{R} \times \mathbf{R}^{d-1}: \xi_{0}+\eta_{0} \cdot y^{*} \leqslant g_{0}^{l}\left(y^{*}\right), y^{*} \in C_{0}\right\} \\
& \mathbf{H}_{+}\left(\xi_{T}, \eta_{T}\right)=\left\{\left(\xi_{0}, \eta_{0}\right) \in \mathbf{R} \times \mathbf{R}^{d-1}: \xi_{0}+\eta_{0} \cdot y^{*} \geqslant g_{0}^{u}\left(y^{*}\right), y^{*} \in C_{0}\right\} .
\end{aligned}
$$

В предположении, что начальный портфель содержит только банковский счет, верхнюю и нижнюю цены хеджирования в рассматриваемой модели естественно определить следующим образом:

$$
\begin{aligned}
& \mathbf{C}^{*}\left(\xi_{T}, \eta_{T}\right)=\inf \left\{\xi_{0}:\left(\xi_{0}, 0\right) \in \mathbf{H}_{+}\right\}=\sup \left\{g_{0}^{u}\left(y^{*}\right): y^{*} \in C_{0}\right\} \\
& \mathbf{C}_{*}\left(\xi_{T}, \eta_{T}\right)=\sup \left\{\xi_{0}:\left(\xi_{0}, 0\right) \in \mathbf{H}_{-}\right\}=\inf \left\{g_{0}^{l}\left(y^{*}\right): y^{*} \in C_{0}\right\}
\end{aligned}
$$

Приведенные выше результаты имеют достаточно прозрачный экономический смысл. Для модели идеального рынка (или рынка без трения) функцию $w_{t}^{u}$, введенную в теореме 1 , можно рассматривать как верхнюю цену (цену продавца) платежного обязательства $f_{T}$ в момент времени $t$. При $t=T$ эта цена равна выплате $f_{T}$. Если цена $w_{t+1}^{u}$ определена, то $w_{t}^{u}$ определяется как минимальная цена $\alpha+\beta \cdot S_{t}$ портфеля $(\alpha, \beta)$, который позволяет с условной вероятностью 1 расплатиться по платежному обязательству $w_{t+1}^{u}$ в момент времени $t+1$.

Для модели рынка с трением при наличии банковского счета (теорема 3 ) функции $g_{t}^{u}$ имеют тот же смысл, но зависят от дополнительного параметра $y^{*}$, который описывает возможные цены активов на «виртуальном» рынке без трения. Эти цены расположены между «реальными» ценами покупки и продажи: $y^{*} \in\left[S_{t}^{2, b}, S_{t}^{2, a}\right] \times \cdots \times\left[S_{t}^{d, b}, S_{t}^{d, a}\right]$, и при определении верхней цены $g_{t}^{u}$ инвестор ориентируется на худший вариант в момент времени $t+1$.

В общей модели рынка с трением (теорема 2) функции $h_{t}^{u}$ дают «инвариантное» описание верхней цены платежного обязательства 
$\left(\zeta_{T}^{1}, \ldots, \zeta_{T}^{d}\right)$ в момент времени $t$, в том смысле, что эти функции зависят только от матрицы $\pi^{i j}$ обменных курсов. Векторы $x^{*} \in K_{t}^{*}$ описывают цены на виртуальном рынке без трения, согласованные с данными обменными курсами: $x^{*, j} / x^{*, i} \in\left[1 / \pi_{t}^{j i}, \pi_{t}^{i j}\right]$.

2. Субдифференциальное описание множеств $\mathbf{H}_{+}, \mathbf{H}_{-}$. Прежде всего сформулируем теорему о мартингальном выборе [15], [17], на которой основаны дальнейшие рассуждения.

Случайный вектор $\eta$ называется селектором $G$, если $\eta(\omega) \in G(\omega)$ для всех $\omega \in \operatorname{dom} G:=\left\{\omega^{\prime}: G\left(\omega^{\prime}\right) \neq \varnothing\right\}$. Последовательность $\left(\eta_{i}\right)_{i=1}^{\infty}$ $\mathscr{F}$-измеримых селекторов $G$ таких, что множества $\left\{\eta_{i}(\omega)\right\}_{i=1}^{\infty}$ плотны в $G(\omega)$ при каждом $\omega \in \operatorname{dom} G$, называется представлением Кастэна для $G$. Известно, что для $\mathscr{F}$-измеримости многозначного отображения с непустыми замкнутыми значениями необходимо и достаточно существования $\mathscr{F}$-измеримого представления Кастэна (см. [14, теорема 14.5]).

$\mathrm{C}$ помощью $\mathrm{cl}$ и conv далее обозначаются замыкание и выпуклая оболочка подмножества конечномерного пространства. Рассмотрим представление Кастэна $\left(\eta_{i}\right)_{i=1}^{\infty}$ для $\mathscr{F}$-измеримого многозначного отображения $G$ с п.н. непустыми замкнутыми значениями $G(\omega)$ и, следуя [15], положим

$$
\varkappa(G, \mathscr{H} ; \omega)=\operatorname{cl}\left(\bigcup_{i=1}^{\infty} \varkappa\left(\eta_{i}, \mathscr{H} ; \omega\right)\right)
$$

где $\varkappa\left(\eta_{i}, \mathscr{H}\right)$ - носитель регулярного условного распределения $\eta_{i}$ относительно $\sigma$-алгебры $\mathscr{H} \subset \mathscr{F}$. В работе [15] показано, что $\mathscr{H}$-измеримое многозначное отображение $\omega \mapsto \varkappa(G, \mathscr{H} ; \omega)$ не зависит от выбора представления Кастэна с точностью до множества нулевой меры. Если $G=\varnothing$ на иножестве положительной меры, то полагаем $\varkappa(G, \mathscr{H})=\varnothing$.

Пусть теперь имеется последовательность $\mathscr{F}_{t}$-измеримых многозначных отображений $G_{t}, t=0, \ldots, T$, с непустыми выпуклыми значениями $G_{t}(\omega) \subset \mathbf{R}^{d}$. Следуя [15], [17], будем говорить, что для последовательности $\left(G_{t}\right)_{t=0}^{T}$ разрешима задача о мартингальном выборе, если существуют согласованный с $\mathbf{F}$ случайный процесс $S_{t} \in \operatorname{ri} G_{t}$ и вероятностная мера $\mathbf{Q} \sim \mathbf{P}$ такие, что $S$ является $\mathbf{Q}$-мартингалом. Введем последовательность многозначных отображений $W_{t}$ по рекуррентной формуле

$$
W_{T}=\operatorname{cl} G_{T}
$$

$$
W_{t}=\operatorname{cl}\left(\operatorname{ri} G_{t} \cap \operatorname{ri} Y_{t}\right), \quad Y_{t}=\operatorname{cl}\left(\operatorname{conv} \varkappa\left(W_{t+1}, \mathscr{F}_{t}\right)\right), \quad 0 \leqslant t \leqslant T-1 .
$$

Данная последовательность корректно определена и согласована с фильтрацией (см. [17]). Заметим, что если $W_{t}=\varnothing$ на множестве положительной меры, то $W_{j}=\varnothing$ при всех $j<t$. 
Теорема 4 (о мартингальном выборе). Для согласованной с фильтрачией последовательности $\left(G_{t}\right)_{t=0}^{T}$ многозначных отображений с непустылм выпукльми значениями $G_{t}(\omega)$ задача о мартингальном выборе разрешима тогда и только тогда, когда все элементь $W_{t}$ последовательности (2.1), (2.2) принимают непустые значения п.н.

В полной общности теорема 4 доказана в работе [17]. Если множества $G_{t}(\omega)$ являются конусами, то разрешимость задачи о мартингальном выборе равносильна существованию $\mathbf{P}$-мартингала $S$, удовлетворяющего условию $S_{t} \in \operatorname{ri} G_{t}$ (см. [15, введение]). Из этого замечания и схемы доказательства теоремы 4 легко вытекает следующий результат.

Лемма 1. Рассмотрим согласованную с фильтрацией последовательность $\left(G_{t}\right)_{t=0}^{T}$ многозначных отображений с непустьли выпукльими коническими значениями $G_{t}(\omega)$. Для $x \in \mathbf{R}^{d}$ существует прочесс $Y \in \mathscr{M}(\operatorname{ri} G)$, удовлетворяюший условию $Y_{0}=x$, тогда и только тогда, когда $x \in \operatorname{ri} W_{0}$.

Заметим, что аналогичное утверждение сформулировано в работе [18].

Зафиксируем платежное обязательство $\zeta_{T} \in L^{0}\left(\mathscr{F}_{T}, \mathbf{R}^{d}\right)$ и введем согласованный с фильтрацией многозначный случайный процесс

$$
U_{t}^{*}=K_{t}^{*} \times \mathbf{R}, \quad 0 \leqslant t<T ; \quad U_{T}^{*}=\left\{\left(x^{*}, y^{*}\right): x^{*} \in K_{T}^{*}, y^{*}=\zeta_{T} \cdot x^{*}\right\},
$$

где $K_{t}$ - конусы платежеспособности. Заметим, что

$$
\begin{aligned}
& \operatorname{ri} U_{t}^{*}=\operatorname{ri} K_{t}^{*} \times \mathbf{R}, \quad 0 \leqslant t<T ; \\
& \operatorname{ri} U_{T}^{*}=\left\{\left(x^{*}, y^{*}\right): x^{*} \in \operatorname{ri} K_{T}^{*}, y^{*}=\zeta_{T} \cdot x^{*}\right\} .
\end{aligned}
$$

Для многозначного случайного процесса с коническими значениями $G_{t}^{*}(\omega)$ положим

$$
\begin{gathered}
\mathscr{W}_{T}^{G}=: G_{T}^{*}, \\
\mathscr{W}_{t}^{G}=:\left(\operatorname{ri} G_{t}^{*} \cap \operatorname{ri} \mathscr{Y}_{t}^{G}\right), \quad \mathscr{Y}_{t}^{G}=:\left(\operatorname{conv} \varkappa\left(\mathscr{W}_{t+1}^{G}, \mathscr{F}_{t}\right)\right), \quad 0 \leqslant t \leqslant T-1 .
\end{gathered}
$$

Лемма 2. Пусть в модели валютного рынка с трением выполнено условие $\mathrm{NA}^{r}$. Тогда для любого платежного обязательства $\zeta_{T} \in L^{0}\left(\mathscr{F}_{T}, \mathbf{R}^{d}\right)$ и любого $\zeta_{0} \in \mathbf{R}^{d}$ следуюшие условия эквивалентны:

(a) $\zeta_{0} \cdot Z_{0}=\mathbf{E}\left(\zeta_{T} \cdot Z_{T}\right)$ для некоторого $Z \in \mathscr{M}\left(\operatorname{ri~} K^{*}, \zeta_{T}\right)$;

(b) существует элемент $x^{*} \in \operatorname{ri} \mathscr{W}_{0}^{K}$ такой, что $\left(x^{*}, \zeta_{0} \cdot x^{*}\right) \in \operatorname{ri} \mathscr{W}_{0}^{U}$ (в обозначениях (2.3), (2.4)).

Д о к а з а т е л ь с т в о. (a) $\Rightarrow(\mathrm{b})$. При выполнении условия (a) положим $y_{t}=\mathbf{E}\left(\zeta_{T} \cdot Z_{T} \mid \mathscr{F}_{t}\right)$. Поскольку $Z \in \mathscr{M}\left(\operatorname{ri} K^{*}\right)$, то $(Z, y) \in \mathscr{M}\left(\operatorname{ri} U^{*}\right)$ и по лемме 1

$$
\left(Z_{0}, y_{0}\right)=\left(Z_{0}, \zeta_{0} \cdot Z_{0}\right) \in \operatorname{ri} \mathscr{W}_{0}^{U}, \quad Z_{0} \in \operatorname{ri} \mathscr{W}_{0}^{K}
$$


(b) $\Rightarrow(a)$. По лемме 1 при выполнении условия (b) существует $(Z, y) \in \mathscr{M}\left(\operatorname{ri} U^{*}\right)$ такой, что

$$
\left(Z_{0}, y_{0}\right)=\left(x^{*}, \zeta_{0} \cdot x^{*}\right) \in \operatorname{ri} \mathscr{W}_{0}^{U} .
$$

При этом $y_{0}=\zeta_{0} \cdot Z_{0}=\mathbf{E} y_{T}=\mathbf{E}\left(\zeta_{T} \cdot Z_{T}\right)$. Лемма доказана.

Для $(x, y) \in \mathbf{R}^{d} \times \mathbf{R}$ положим $\operatorname{Pr}(x, y)=x$. В следующей лемме устанавливается связь между процессами $\mathscr{W}^{U}$ и $\mathscr{W}^{K}$.

Лемма 3. Справедливы равенства $\operatorname{ri} \mathscr{W}_{t}^{K}=\operatorname{Pr}\left(\operatorname{ri} \mathscr{W}_{t}^{U}\right)$ n.н., $t=$ $0, \ldots, T$.

Д о к а з а т е л ь с т в о. Из определения $U_{T}^{*}$ следует, что

$$
\operatorname{Pr} \mathscr{W}_{T}^{U}=\operatorname{Pr} U_{T}^{*}=K_{T}^{*}=\mathscr{W}_{T}^{K} .
$$

Предположим, что ri $\mathscr{W}_{t+1}^{K}=\operatorname{Pr}\left(\operatorname{ri} \mathscr{W}_{t+1}^{U}\right)$. Интересен лишь случай $\mathscr{W}_{t+1}^{K} \neq \varnothing$ п.н. Пусть $\left(\xi_{t+1}^{i}\right)_{i=1}^{\infty}-\mathscr{F}_{t+1}$-измеримое представление Кастэна для $\mathscr{W}_{t+1}^{U}$. Тогда $\left(\eta_{t+1}^{i}\right)_{i=1}^{\infty}, \eta_{t+1}^{i}=\operatorname{Pr} \xi_{t+1}^{i},-$ представление Кастэна для $\operatorname{cl}\left(\operatorname{Pr} \mathscr{W}_{t+1}^{U}\right)=\mathscr{W}_{t+1}^{K}$. Пусть $\mathbf{P}_{\eta_{t+1}}$ - регулярное условное распределение $\eta_{t+1}$ относительно $\mathscr{F}_{t}$. Если $\eta_{t+1}=\operatorname{Pr} \xi_{t+1}$, то

$$
\varkappa\left(\eta_{t+1}, \mathscr{F}_{t}\right)=\operatorname{cl}\left(\operatorname{Pr} \varkappa\left(\xi_{t+1}, \mathscr{F}_{t}\right)\right) \text { п.н. }
$$

Чтобы доказать это утверждение, заметим сначала, что

$$
\begin{aligned}
\mathbf{P}_{\eta_{t+1}}(\omega, B) & =\mathbf{P}\left(\eta_{t+1} \in B \mid \mathscr{F}_{t}\right)(\omega)=\mathbf{P}\left(\xi_{t+1} \in B \times \mathbf{R} \mid \mathscr{F}_{t}\right)(\omega) \\
& =\mathbf{P}_{\xi_{t+1}}(\omega, B \times \mathbf{R}) \quad \text { п.н., } \quad B \in \mathscr{B}\left(\mathbf{R}^{d}\right) .
\end{aligned}
$$

Пусть $\mathbf{P}_{\eta_{t+1}}(\omega, V)=\mathbf{P}_{\xi_{t+1}}(\omega, V \times \mathbf{R})$ для всех $\omega \in \Omega^{\prime}$, где $\mathbf{P}\left(\Omega^{\prime}\right)=1$, и всех $V$ из некоторой счетной базы $\mathscr{V}$ открытых множеств $\mathbf{R}^{d}$. Если $\omega \in \Omega^{\prime}$ и точка $(x, y) \in \mathbf{R}^{d} \times \mathbf{R}$ принадлежит $\varkappa\left(\xi_{t+1}, \mathscr{F}_{t}\right)(\omega)$, то

$$
\mathbf{P}_{\eta_{t+1}}\left(\omega, V_{x}\right)=\mathbf{P}_{\xi_{t+1}}\left(\omega, V_{x} \times \mathbf{R}\right)>0
$$

для любой окрестности $V_{x} \in \mathscr{V}$ точки $x$. Следовательно, $x \in \varkappa\left(\eta_{t+1}, \mathscr{F}_{t}\right)$.

С другой стороны, для $x \in \varkappa\left(\eta_{t+1}, \mathscr{F}_{t}\right)(\omega), \omega \in \Omega^{\prime}$, также выполняются соотношения (2.6). Следовательно, множество $\left(V_{x} \times \mathbf{R}\right) \cap \varkappa\left(\xi_{t+1}, \mathscr{F}_{t}\right)$ непусто и содержит точку $\left(x^{\prime}, y^{\prime}\right)$, проекция которой принадлежит $V_{x}$.

Итак, доказано, что

$$
\operatorname{Pr} \varkappa\left(\xi_{t+1}, \mathscr{F}_{t}\right) \subset \varkappa\left(\eta_{t+1}, \mathscr{F}_{t}\right) \subset \operatorname{cl}\left(\operatorname{Pr} \varkappa\left(\xi_{t+1}, \mathscr{F}_{t}\right)\right) \text { п.н. }
$$

Отсюда вытекает (2.5).

Пусть $B, B_{i}$ - любые подмножества $\mathbf{R}^{d+1}$. Используя элементарные соотношения $\operatorname{cl}\left(\bigcup_{i=1}^{\infty} \operatorname{cl} B_{i}\right)=\operatorname{cl}\left(\bigcup_{i=1}^{\infty} B_{i}\right), \operatorname{ri}(\operatorname{conv}(\operatorname{cl} B))=\operatorname{ri}(\operatorname{conv} B)$ и 
формулу (2.5), находим

$$
\begin{aligned}
\varkappa\left(\mathscr{W}_{t+1}^{K}, \mathscr{F}_{t}\right) & =\operatorname{cl}\left(\bigcup_{i=1}^{\infty} \varkappa\left(\eta_{t+1}^{i}, \mathscr{F}_{t}\right)\right)=\operatorname{cl}\left(\bigcup_{i=1}^{\infty} \operatorname{cl}\left(\operatorname{Pr} \varkappa\left(\xi_{t+1}^{i}, \mathscr{F}_{t}\right)\right)\right) \\
& =\operatorname{cl}\left(\operatorname{Pr}\left(\bigcup_{i=1}^{\infty} \varkappa\left(\xi_{t+1}^{i}, \mathscr{F}_{t}\right)\right)\right)=\operatorname{cl}\left(\operatorname{Pr} \varkappa\left(\mathscr{W}_{t+1}^{U}, \mathscr{F}_{t}\right)\right) \quad \text { п.н.; } \\
\operatorname{ri} \mathscr{Y}_{t}^{K} & =\operatorname{ri}\left(\operatorname{conv} \varkappa\left(\mathscr{W}_{t+1}^{K}, \mathscr{F}_{t}\right)\right)=\operatorname{ri}\left(\operatorname{conv}\left(\operatorname{cl}\left(\operatorname{Pr} \varkappa\left(\mathscr{W}_{t+1}^{U}, \mathscr{F}_{t}\right)\right)\right)\right) \\
& =\operatorname{ri}\left(\operatorname{Pr}\left(\operatorname{conv} \varkappa\left(\mathscr{W}_{t+1}^{U}, \mathscr{F}_{t}\right)\right)\right)=\operatorname{Pr}\left(\operatorname{ri} \mathscr{Y}_{t}^{U}\right) \quad \text { п.н. }
\end{aligned}
$$

Доказательство леммы 3 завершает следующая цепочка равенств:

$$
\begin{aligned}
\operatorname{ri} \mathscr{W}_{t}^{K} & =\operatorname{ri} K_{t}^{*} \cap \operatorname{ri} \mathscr{Y}_{t}^{K}=\operatorname{Pr}\left(\operatorname{ri}\left(K_{t}^{*} \times \mathbf{R}\right)\right) \cap \operatorname{Pr}\left(\operatorname{ri} \mathscr{Y}_{t}^{U}\right) \\
& =\operatorname{Pr}\left(\operatorname{ri} U_{t}^{*} \cap \operatorname{ri} \mathscr{Y}_{t}^{U}\right)=\operatorname{Pr}\left(\operatorname{ri} \mathscr{W}_{t}^{U}\right) \quad \text { п.н. }
\end{aligned}
$$

Следующая лемма показывает, что функции, которые определяются формулами

$$
\varphi_{t}^{l}\left(x^{*}\right)=\inf \left\{y^{*}:\left(x^{*}, y^{*}\right) \in \mathscr{Y}_{t}^{U}\right\}, \quad \psi_{t}^{l}\left(x^{*}\right)=\inf \left\{y^{*}:\left(x^{*}, y^{*}\right) \in \mathscr{W}_{t}^{U}\right\},
$$

являются положительно однородными, выпуклыми и замкнутыми. Напомним, что расширенная выпуклая функция $f$ называется собственной, если ее надграфик непуст и не содержит вертикальных прямых: [13, гл. $1, \S 4]$.

Лемма 4. Для замкнутого выпуклого конуса $A \subset \mathbf{R}^{d} \times \mathbf{R}$ функиия

$$
\psi(x)=\inf \{y:(x, y) \in A\}
$$

положительно однородна, выпукла и замкнута. Она является собственной тогда и только тогда, когда

$$
\left\{x^{\prime} \in \mathbf{R}^{d}:\left(-x^{\prime}, 1\right) \in A^{*}\right\} \neq \varnothing .
$$

Если $\psi$ собственная, то условие $z \in \partial \psi$ равносильно неравенству

$$
x \cdot z \leqslant \psi(x), \quad x \in \operatorname{ri}(\operatorname{Pr} A)
$$

и имеет место представление

$$
\psi(x)=\sup \left\{x \cdot x^{\prime}:\left(-x^{\prime}, 1\right) \in A^{*}\right\} .
$$

Если $\psi$ несобственная, то

$$
\psi(x)=-\infty, \quad x \in \operatorname{Pr} A ; \quad \psi(x)=+\infty, \quad x \notin \operatorname{Pr} A .
$$


Д о к а з а т е л ь с т в о. Для любых $\left(x_{i}, z_{i}\right) \in$ epi $\psi, i=1,2$, существуют $y_{i} \leqslant z_{i}$ такие, что $\left(x_{i}, y_{i}\right) \in A, i=1,2$. В силу выпуклости $A$ имеем

$$
\left(\alpha_{1} x_{1}+\alpha_{2} x_{2}, \alpha_{1} y_{1}+\alpha_{2} y_{2}\right) \in A,
$$

где $\alpha_{1}+\alpha_{2}=1, \alpha_{1}, \alpha_{2} \geqslant 0$. Далее, поскольку $\alpha_{1} y_{1}+\alpha_{2} y_{2} \leqslant \alpha_{1} z_{1}+\alpha_{2} z_{2}$, то $\psi$ является выпуклой: $\left(\alpha_{1} x_{1}+\alpha_{2} x_{2}, \alpha_{1} z_{1}+\alpha_{2} z_{2}\right) \in$ epi $\psi$.

Рассмотрим последовательность $\left(x_{k}, z_{k}\right) \in$ eрі $\psi, k \in \mathbf{N}$, сходящуюся к $(x, z)$. Существуют $y_{k} \leqslant z_{k}$ такие, что $\left(x_{k}, y_{k}\right) \in A$. Выберем подпоследовательность $\left(y_{k_{n}}\right)$, сходящуюся к $y$. В силу замкнутости $A$ имеем

$$
\left(x_{k_{n}}, y_{k_{n}}\right) \rightarrow(x, y) \in A, \quad y \leqslant z .
$$

Значит, множество ері $\psi$ замкнуто: $(x, z) \in$ ері $\psi$.

Положительная однородность $\psi$ очевидна:

$$
\psi(\lambda x)=\inf \{y:(\lambda x, y) \in A\}=\inf \{\lambda y:(\lambda x, \lambda y) \in A\}=\lambda \psi(x), \quad \lambda>0 .
$$

Если условие (2.8) выполнено, то существует $x^{\prime} \in \mathbf{R}^{d}$ такой, что $y \geqslant x \cdot x^{\prime},(x, y) \in A$. Следовательно, $\psi(x)>-\infty, x \in A$, и $\psi$ является собственной. Пусть $\psi-$ собственная функция. Найдем ее вторую сопряженную по Юнгу-Фенхелю:

$$
\begin{aligned}
& \psi^{*}\left(x^{\prime}\right)=\sup _{x}\left\{x \cdot x^{\prime}-\psi(x)\right\}=\sup \left\{x \cdot x^{\prime}-y:(x, y) \in A\right\}=\delta\left(\left(-x^{\prime}, 1\right) \mid A^{*}\right), \\
& \psi^{* *}(x)=\sup _{x^{\prime}}\left\{x \cdot x^{\prime}-\psi^{*}\left(x^{\prime}\right)\right\}=\sup \left\{x \cdot x^{\prime}:\left(-x^{\prime}, 1\right) \in A^{*}\right\} .
\end{aligned}
$$

Поскольку $\psi$ является выпуклой и замкнутой, то $\psi=\psi^{* *}$ (см. [13, теорема 12.2]). Отсюда вытекает (2.8) и представление (2.10).

Ясно, что $\operatorname{Pr}($ epi $\psi)=\operatorname{Pr} A$, если $\psi$ является собственной. Далее, множество ri (epi $\psi)$ состоит из пар $(x, y)$, для которых $x \in \operatorname{ri}(\operatorname{Pr}(\operatorname{epi} \psi))$, $\psi(x)<y$ (см. [13, лемма 7.3]), и из неравенства (2.9) вытекает, что $x \cdot z \leqslant y$ для всех таких пар. Отсюда очевидным образом следует, что $z \in \partial \psi$.

Соотношения (2.11) вытекают из того, что несобственная замкнутая выпуклая функция $\psi$ не может принимать конечных значений (см. [13], следствие 7.2.1). Лемма доказана.

Введем функции

$$
\varphi_{t}^{u}\left(x^{*}\right)=\sup \left\{y^{*}:\left(x^{*}, y^{*}\right) \in \mathscr{Y}_{t}^{U}\right\}, \quad \psi_{t}^{u}\left(x^{*}\right)=\sup \left\{y^{*}:\left(x^{*}, y^{*}\right) \in \mathscr{W}_{t}^{U}\right\} .
$$

Ясно, что $-\varphi_{t}^{u},-\psi_{t}^{u}$ положительно однородны, выпуклы и замкнуты. Из теоремы 4 и леммы 3 вытекает разрешимость задачи о мартингальном выборе для процесса $U^{*}$ при выполнении условия $\mathrm{NA}^{r}$. При этом ri $\mathscr{W}_{t}^{U}=$ $\operatorname{ri}\left(K_{t}^{*} \times \mathbf{R}\right) \cap \operatorname{ri} \mathscr{Y}_{t}^{U} \neq \varnothing$ п.н. и

$$
\mathscr{W}_{t}^{U}=\left(K_{t}^{*} \times \mathbf{R}\right) \cap \mathscr{Y}_{t}^{U} \text { п.н. }
$$


Таким образом, $\psi_{t}^{l}\left(x^{*}\right)=\varphi_{t}^{l}\left(x^{*}\right)+\delta\left(x^{*} \mid K_{t}^{*}\right), \psi_{t}^{u}\left(x^{*}\right)=\varphi_{t}^{u}\left(x^{*}\right)-\delta\left(x^{*} \mid K_{t}^{*}\right)$.

Заметим, что

$\left\{y^{*}:\left(x^{*}, y^{*}\right) \in \mathscr{W}_{t}^{U}\right\}=\left\{y^{*}: y^{*} \in\left[\psi_{t}^{l}\left(x^{*}\right), \psi_{t}^{u}\left(x^{*}\right)\right] \cap \mathbf{R}\right\}, \quad 0 \leqslant t \leqslant T$.

Поскольку $\operatorname{ri}\left(\operatorname{Pr} \mathscr{W}_{t}^{U}\right)=\operatorname{ri} \mathscr{W}_{t}^{K}$ по лемме 3 , то в силу теоремы 6.8 из [13] имеем

$$
\operatorname{ri} \mathscr{W}_{t}^{U}=\left\{\left(x^{*}, y^{*}\right): x^{*} \in \operatorname{ri} \mathscr{W}_{t}^{K}, y^{*} \in \operatorname{ri}\left(\left[\psi_{t}^{l}\left(x^{*}\right), \psi_{t}^{u}\left(x^{*}\right)\right] \cap \mathbf{R}\right)\right\} .
$$

Пусть $\mathbf{J}\left(\zeta_{T}\right)$ обозначает множество элементов $\zeta_{0} \in \mathbf{R}^{d}$, удовлетворяющих условию (a) леммы 2. Согласно условию (b) указанной леммы $\zeta_{0} \in \mathbf{J}\left(\zeta_{T}\right)$, тогда и только тогда, когда существует $x^{*} \in \operatorname{ri} \mathscr{W}_{0}^{K}$ такой, что

$$
\zeta_{0} \cdot x^{*} \in \operatorname{ri}\left(\left[\psi_{0}^{l}\left(x^{*}\right), \psi_{0}^{u}\left(x^{*}\right)\right] \cap \mathbf{R}\right) .
$$

Теорема 5. При выполнении условия $\mathrm{NA}^{r}$ справедливы равенства

$$
\mathbf{H}_{-}\left(\zeta_{T}\right)=\partial \psi_{0}^{l}, \quad \mathbf{H}_{+}\left(\zeta_{T}\right)=-\partial\left(-\psi_{0}^{u}\right) .
$$

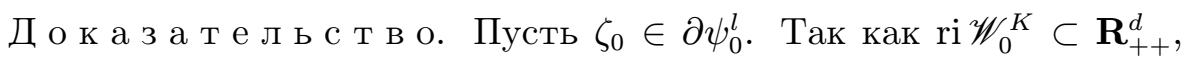
To

$$
\left(\zeta_{0}-a\right) \cdot x^{*}<\psi_{0}^{l}\left(x^{*}\right), \quad x^{*} \in \mathrm{ri} \mathscr{W}_{0}^{K}, \quad a \in \mathbf{R}_{++}^{d} .
$$

Следовательно, $\zeta_{0}-a \notin \mathbf{J}\left(\zeta_{T}\right)$, т.е.

$$
\left(\zeta_{0}-a\right) \cdot Z_{0} \neq \mathbf{E}\left(\zeta_{T} \cdot Z_{T}\right), \quad Z \in \mathscr{M}\left(\mathrm{ri} K^{*}, \zeta_{T}\right) .
$$

С другой стороны, для любого $x^{\prime} \in \mathrm{ri} \mathscr{W}_{0}^{K}$ существует элемент $b \in$ $\mathbf{R}_{+}^{d}$ такой, что

$$
\left(\zeta_{0}+b\right) \cdot x^{\prime} \in \operatorname{ri}\left(\left[\psi_{0}^{l}\left(x^{\prime}\right), \psi_{0}^{u}\left(x^{\prime}\right)\right] \cap \mathbf{R}\right) .
$$

При этом $\zeta_{0}+b \in \mathbf{J}\left(\zeta_{T}\right)$ и существует $Z^{\prime} \in \mathscr{M}\left(\mathrm{ri} K^{*}, \zeta_{T}\right)$, удовлетворяющий соотношениям

$$
\left(\zeta_{0}-a\right) \cdot Z_{0}^{\prime}<\left(\zeta_{0}+b\right) \cdot Z_{0}^{\prime}=\mathbf{E}\left(\zeta_{T} \cdot Z_{T}^{\prime}\right) .
$$

Если предположить, что $\left(\zeta_{0}-a\right) \cdot Z_{0}^{\prime \prime}>\mathbf{E}\left(\zeta_{T} \cdot Z_{T}^{\prime \prime}\right)$ для некоторого $Z^{\prime \prime} \in \mathscr{M}\left(\right.$ ri $\left.K^{*}, \zeta_{T}\right)$, то найдется $\alpha \in(0,1)$ такое, что

$$
\left(\zeta_{0}-a\right) \cdot Z_{0}^{\alpha}=\mathbf{E}\left(\zeta_{T} \cdot Z_{T}^{\alpha}\right),
$$

где $Z^{\alpha}=\alpha Z^{\prime}+(1-\alpha) Z^{\prime \prime}$. Но это противоречит (2.14). Следовательно, $\zeta_{0}-a \in \mathbf{H}_{-}$(см. (1.4)) и $\zeta_{0} \in \mathbf{H}_{-}$в силу замкнутости $\mathbf{H}_{-}$.

Пусть теперь $\zeta_{0} \in \mathbf{H}_{-}$. Тогда для любого $a \in \mathbf{R}_{++}^{d}$ имеем

$$
\left(\zeta_{0}-a\right) \cdot Z_{0}<\zeta_{0} \cdot Z_{0} \leqslant \mathbf{E}\left(\zeta_{T} \cdot Z_{T}\right), \quad Z \in \mathscr{M}\left(\text { ri } K^{*}, \zeta_{T}\right) .
$$


Следовательно, $\zeta_{0}-a \notin \mathbf{J}\left(\zeta_{T}\right)$. По лемме 2 отсюда следует, что

$$
\left(\zeta_{0}-a\right) \cdot x^{*} \notin \operatorname{ri}\left(\left[\psi_{0}^{l}\left(x^{*}\right), \psi_{0}^{u}\left(x^{*}\right)\right] \cap \mathbf{R}\right)
$$

для любого $x^{*} \in \mathrm{ri} \mathscr{W}_{0}^{K}$. Более того, не существует такой пары $x^{\prime}, x^{\prime \prime} \in$ ri $\mathscr{W}_{0}^{K}$, что

$$
\left(\zeta_{0}-a\right) \cdot x^{\prime} \leqslant \psi_{0}^{l}\left(x^{\prime}\right), \quad\left(\zeta_{0}-a\right) \cdot x^{\prime \prime} \geqslant \psi_{0}^{u}\left(x^{\prime \prime}\right) .
$$

Действительно, пусть указанная пара $x^{\prime}, x^{\prime \prime}$ существует. Тогда $\psi_{0}^{l}$, $-\psi_{0}^{u}$ являются собственными и их значения на ri $\mathscr{W}_{0}^{K}$ конечны. Для любой непрерывной функции $\beta:[0,1] \rightarrow(0,1)$ имеем

$$
\begin{aligned}
& x^{\prime} \cdot\left(\zeta_{0}-a\right)<\beta(1) \psi_{0}^{l}\left(x^{\prime}\right)+(1-\beta(1)) \psi_{0}^{u}\left(x^{\prime}\right) \in \operatorname{ri}\left[\psi_{0}^{l}\left(x^{\prime}\right), \psi_{0}^{u}\left(x^{\prime}\right)\right], \\
& x^{\prime \prime} \cdot\left(\zeta_{0}-a\right)>\beta(0) \psi_{0}^{l}\left(x^{\prime \prime}\right)+(1-\beta(0)) \psi_{0}^{u}\left(x^{\prime \prime}\right) \in \operatorname{ri}\left[\psi_{0}^{l}\left(x^{\prime \prime}\right), \psi_{0}^{u}\left(x^{\prime \prime}\right)\right]
\end{aligned}
$$

и существует $\alpha \in(0,1)$, для которого справедливо равенство

$$
\begin{aligned}
\left(\alpha x^{\prime}+(1-\alpha) x^{\prime \prime}\right) \cdot\left(\zeta_{0}-a\right)= & \beta(\alpha) \psi_{0}^{l}\left(\alpha x^{\prime}+(1-\alpha) x^{\prime \prime}\right) \\
& +(1-\beta(\alpha)) \psi_{0}^{u}\left(\alpha x^{\prime}+(1-\alpha) x^{\prime \prime}\right) .
\end{aligned}
$$

Но существование такой точки $\alpha x^{\prime}+(1-\alpha) x^{\prime \prime} \in$ ri $\mathscr{W}_{0}^{K}$ противоречит (2.16).

Далее, для любого $Z^{\prime} \in \mathscr{M}\left(\right.$ ri $\left.K^{*}, \zeta_{T}\right)$ существует элемент $b \in \mathbf{R}_{+}^{d}$ такой, что $\left(\zeta_{0}+b\right) \cdot Z_{0}^{\prime}=\mathbf{E}\left(\zeta_{T} \cdot Z_{T}^{\prime}\right)$. Значит, $\zeta_{0}+b \in \mathbf{J}\left(\zeta_{T}\right)$ и существует $x^{\prime} \in \mathrm{ri} \mathscr{W}_{0}^{K}$, удовлетворяющий (2.15). Таким образом,

$$
\left(\zeta_{0}-a, x^{\prime}\right)<\left(\zeta_{0}+b, x^{\prime}\right) \leqslant \psi_{0}^{u}\left(x^{\prime}\right) .
$$

Как показано выше, отсюда следует, что первое соотношение (2.17) выполняется для всех $x^{\prime} \in \operatorname{ri} \mathscr{W}_{0}^{K}$. Это означает, что $\zeta_{0}-a \in \partial \psi_{0}^{l}$ и $\zeta_{0} \in \partial \psi_{0}^{l}$ в силу замкнутости $\partial \psi_{0}^{l}$.

Второе равенство (2.13) устанавливается аналогичным образом.

3. Рекуррентные формулы для $\mathbf{H}_{+}, \mathbf{H}_{-}$. Пусть $\mathscr{R}$ обозначает множество всех расширенных функций на $\mathbf{R}^{d}$ с естественным упорядочением: $f \leqslant \mathscr{R} \psi$, если $f(x) \leqslant \psi(x)$ для всех $x \in \mathbf{R}^{d}$. Будем говорить, что интегранд $\psi$ является существенным $\mathscr{R}$-супремумом семейства $\Psi=\left\{\psi^{\alpha}\right\}_{\alpha \in I}$ интеграндов, если

1) $\psi(\omega, \cdot) \geqslant \mathscr{R} \psi^{\alpha}(\omega, \cdot)$ п.н.;

2) из того, что $\varphi(\omega, \cdot) \geqslant \mathscr{R} \psi^{\alpha}(\omega, \cdot)$ п.н., следует, что и $\varphi(\omega, \cdot) \geqslant \mathscr{R}$ $\psi(\omega, \cdot)$ п.н.

Будем использовать обозначение $\psi=\mathscr{R}$-ess $\sup \left\{\psi_{\alpha}: \alpha \in I\right\}$. Ясно, что существенный $\mathscr{R}$-супремум единственен с точностью до неразличимости.

Напомним, что для любого измеримого многозначного отображения $G$ опорная функция $\sup \{z \cdot x: z \in G(\omega)\}$ является выпуклым нормальным интеграндом [14, пример 14.51]. 
Лемма 5. Пусть многозначное отображение $\omega \mapsto G(\omega) \subset \mathbf{R}^{d}$ является $\mathscr{H}$-измеримьм. Тогда

$$
\mathscr{R}-\operatorname{ess} \sup \left\{\xi \cdot x: \xi \in L^{0}(\mathscr{H}, G)\right\}=\sup \{z \cdot x: z \in G(\omega)\}
$$

с точностью до неразличимости.

Д о к а з а т е л ь с т в о. Далее все неравенства понимаются с точностью до неразличимости. Для любого $\xi \in L^{0}(\mathscr{H}, G)$ имеем

$$
\xi(\omega) \cdot x \leqslant \sup \{z \cdot x: z \in G(\omega)\} .
$$

Поэтому левая часть (3.1) не превосходит правой части. Чтобы получить противоположное неравенство, рассмотрим $\mathscr{H}$-измеримое представление Кастэна $\left(\xi_{n}\right)_{n=1}^{\infty}$ отображения $\mathrm{cl} G$ и заметим, что

$$
\sup \{z \cdot x: z \in G(\omega)\}=\sup _{n}\left(\xi_{n}(\omega) \cdot x\right) \leqslant \mathscr{R}-\operatorname{ess} \sup \left\{\xi \cdot x: \xi \in L^{0}(\mathscr{H}, G)\right\} .
$$

Лемма 6. Пусть выполнено условие $\mathrm{NA}^{r}$. Интегранд $\varphi_{t}^{l}, 0 \leqslant t \leqslant$ $T-1$, определяемый формулой (2.7), является собственным п.н., тогда и только тогда, когда $L^{0}\left(\mathscr{F}_{t}, \partial \psi_{t+1}^{l}\right) \neq \varnothing$. Если $\varphi_{t}^{l}$ является собственнblм п.н., mo

$$
\varphi_{t}^{l}=\mathscr{R}-\operatorname{ess} \sup \left\{\xi \cdot x^{*}: \xi \in L^{0}\left(\mathscr{F}_{t}, \partial \psi_{t+1}^{l}\right)\right\} .
$$

Если $\mathbf{H}_{-}\left(\zeta_{T}\right) \neq \varnothing$, то интегранд $\varphi_{t}^{l}$ является собственным п.н. для вcext.

Д о к а з а т е л ь с т в о. Покажем, что

$$
L^{0}\left(\mathscr{F}_{t}, \partial \psi_{t+1}^{l}\right)=\left\{\xi:(-\xi, 1) \in L^{0}\left(\mathscr{F}_{t},\left(\mathscr{Y}_{t}^{U}\right)^{*}\right)\right\} .
$$

Заметим, что по леммам 3 и 4 для $\mathscr{F}_{t}$-измеримого вектора $\xi$ условие $\xi \in \partial \psi_{t+1}^{l}$ п.н. равносильно следующему:

$$
\xi \cdot x^{\prime} \leqslant \psi_{t+1}^{l}\left(x^{\prime}\right), \quad x^{\prime} \in \operatorname{ri} \mathscr{W}_{t+1}^{K} \quad \text { п.н. }
$$

В свою очередь, (3.4) эквивалентно условию

$$
\xi \cdot x^{\prime}-y^{\prime} \leqslant 0, \quad x^{\prime} \in \operatorname{ri} \mathscr{W}_{t+1}^{K}, \quad y^{\prime} \in \operatorname{ri}\left(\left[\psi_{t+1}^{l}\left(x^{\prime}\right), \psi_{t+1}^{u}\left(x^{\prime}\right)\right] \cap \mathbf{R}\right) \quad \text { п.н. }
$$

которое в силу $(2.12)$ можно представить в виде $(-\xi, 1) \in\left(\mathscr{W}_{t+1}^{U}\right)^{*}$ п.н. Согласно лемме 1 работы [16], имеем

$$
L^{0}\left(\mathscr{F}_{t},\left(\mathscr{W}_{t+1}^{U}\right)^{*}\right)=L^{0}\left(\mathscr{F}_{t},\left(\varkappa\left(\mathscr{W}_{t+1}^{U}, \mathscr{F}_{t}\right)\right)^{*}\right)=L^{0}\left(\mathscr{F}_{t},\left(\mathscr{Y}_{t}^{U}\right)^{*}\right)
$$

Отсюда вытекает (3.3). 
Если интегранд $\varphi_{t}^{l}$ является собственным п.н., то по лемме 4 имеем

$$
\varphi_{t}^{l}\left(\omega, x^{*}\right)=\sup \left\{x \cdot x^{*}:(-x, 1) \in\left(\mathscr{Y}_{t}^{U}\right)^{*}(\omega)\right\} \quad \text { п.н. }
$$

Но тогда по лемме 5

$$
\varphi_{t}^{l}=\mathscr{R}-\operatorname{ess} \sup \left\{\xi \cdot x^{*}:(-\xi, 1) \in L^{0}\left(\mathscr{F}_{t},\left(\mathscr{Y}_{t}^{U}\right)^{*}\right)\right\}
$$

что вместе с (3.3) дает представление (3.2). При этом $L^{0}\left(\mathscr{F}_{t}, \partial \psi_{t+1}^{l}\right) \neq \varnothing$.

Если же $L^{0}\left(\mathscr{F}_{t}, \partial \psi_{t+1}^{l}\right) \neq \varnothing$, то множество $\left\{x:(-x, 1) \in\left(\mathscr{Y}_{t}^{U}\right)^{*}\right\}$ непусто п.н. (в силу $(3.3)$ ), и интегранд $\varphi_{t}^{l}$ является собственным п.н. по лемме 4.

Докажем последнее утверждение леммы 6 . Если $\mathbf{H}_{-} \neq \varnothing$, то $\psi_{0}^{l}$ является собственным по теореме 5. Заметим, что если $\psi_{t}^{l}-$ п.н. собственный интегранд, то и $\varphi_{t}^{l}$ является собственным п.н., так как $\psi_{t}^{l}=\varphi_{t}^{l}$ на $K_{t}^{*}$. По доказанному при этом имеет место равенство $(3.2)$ и, в частности, $\psi_{t+1}^{l}$ является собственным п.н. По индукции отсюда вытекает, что $\varphi_{t}^{l}$ является собственным для всех $t$.

Д ок аз а т е л ь с т в о т е о р е м ы 2. Проведем доказательство для последовательности $\left(h_{t}^{l}\right)_{t=0}^{T}$. Заметим сначала, что

$$
h_{t}^{l}\left(\omega, x^{*}\right)=\mathscr{R}-\operatorname{ess} \sup \left\{\xi \cdot x^{*}: \xi \in L^{0}\left(\mathscr{F}_{t}, G_{t}^{l}\right)\right\}+\delta\left(x^{*} \mid K_{t}^{*}\right), \quad t \leqslant T-1,
$$

по лемме 5. Далее, с учетом соотношения (1.6), для $\mathscr{F}_{t}$-измеримого вектора $\xi$ по определению регулярного условного математического ожидания справедливо равенство

$$
\begin{aligned}
\mathbf{E}^{r}\left(\mathbf{I}\left(z \in \partial h_{t+1}^{l}\right) \mid \mathscr{F}_{t}\right)(\omega, \xi(\omega)) & =\mathbf{E}\left(\mathbf{I}\left(h_{t+1}^{1, *}(\omega, \xi(\omega)) \leqslant 0\right) \mid \mathscr{F}_{t}\right) \\
& =\mathbf{E}\left(\mathbf{I}\left(\xi \in \partial h_{t+1}^{l}\right) \mid \mathscr{F}_{t}\right)(\omega)
\end{aligned}
$$

и по определению $G_{t}^{l}$ имеем

$$
\begin{aligned}
L^{0}\left(\mathscr{F}_{t}, G_{t}^{l}\right) & =\left\{\xi \in L^{0}\left(\mathscr{F}_{t}, \mathbf{R}^{d}\right): \mathbf{E}\left(\mathbf{I}\left(\xi \in \partial h_{t+1}^{l}\right) \mid \mathscr{F}_{t}\right)(\omega)=1 \text { п.н. }\right\} \\
& =\left\{\xi \in L^{0}\left(\mathscr{F}_{t}, \mathbf{R}^{d}\right): \mathbf{P}\left(\xi \in \partial h_{t+1}^{l}\right)=1\right\}=L^{0}\left(\mathscr{F}_{t}, \partial h_{t+1}^{l}\right) .
\end{aligned}
$$
$\psi_{T}^{l}:$

Предположим, что $G_{t}(\omega) \neq \varnothing$ п.н. для всех $t$. По определению $h_{T}^{l}=$

$$
\begin{aligned}
\psi_{T}^{l}\left(x^{*}\right) & =\inf \left\{y^{*}:\left(x^{*}, y^{*}\right) \in U_{T}^{*}\right\}=\inf \left\{y^{*}: x^{*} \in K_{T}^{*}, y^{*}=\zeta_{T} \cdot x^{*}\right\} \\
& =\zeta_{T} \cdot x^{*}+\delta\left(x^{*} \mid K_{T}^{*}\right) .
\end{aligned}
$$

Пусть $h_{t+1}^{l}$ и $\psi_{t+1}^{l}$ неразличимы. Заметим, что $L^{0}\left(\mathscr{F}_{t}, G_{t}^{l}\right) \neq \varnothing$ по теореме об измеримом выборе (см. [14, следствие 14.6]) и, значит, 
$L^{0}\left(\mathscr{F}_{t}, \partial \psi_{t+1}^{l}\right) \neq \varnothing$ в силу (3.6). Из формул (3.5), (3.6) и леммы 6 вытекает, что интегранд $\varphi_{t}^{l}$ является собственным п.н. и

$$
\begin{aligned}
h_{t}^{l}\left(x^{*}\right) & =\mathscr{R}-\operatorname{ess} \sup \left\{\xi \cdot x^{*}: \xi \in L^{0}\left(\mathscr{F}_{t}, \partial \psi_{t+1}^{l}\right)\right\}+\delta\left(x^{*} \mid K_{t}^{*}\right) \\
& =\varphi_{t}^{l}\left(x^{*}\right)+\delta\left(x^{*} \mid K_{t}^{*}\right)=\psi_{t}^{l}\left(x^{*}\right) .
\end{aligned}
$$

По индукции интегранды $h_{t}^{l}, \psi_{t}^{l}$ неразличимы при всех $t$. При этом $\mathbf{H}_{-}=\partial \psi_{0}^{l}=\partial h_{0}^{l}$ по теореме 5 .

Предположим теперь, что $G_{t} \neq \varnothing$ п.н., $t=m+1, \ldots, T-1$, и $G_{m}=\varnothing$ на множестве $A_{m} \in \mathscr{F}_{m}$ положительной меры. Предыдущие рассуждения показывают, что $\psi_{t+1}^{l}$ и $h_{t+1}^{l}$ неразличимы при $t \geqslant m$. Из (3.6) следует, что $L^{0}\left(\partial \psi_{m+1}^{l}, \mathscr{F}_{m}\right)=\varnothing$. По лемме 6 отсюда вытекает, что $\mathbf{H}_{-}=\varnothing$. Теорема доказана.

Рассмотрим модель валютного рынка без операционных издержек, в которой матрица обменных курсов имеет вид $\pi^{i j}=\widehat{S}_{t}^{j} / \widehat{S}_{t}^{i}$, где $\widehat{S}_{t}=$ $\left(\widehat{S}_{t}^{1}, \ldots, \widehat{S}_{t}^{d}\right)$ - согласованный с $\mathbf{F}$ случайный процесс с положительными компонентами. При этом теорема 2 может быть конкретизирована следующим образом.

Теорема 6. Пусть модель валютного рынка без операционных издержек удовлетворяет условию отсутствия арбитража (NA) (равносильному условию $\mathrm{NA}^{r}$ ). Введем согласованные с $\mathbf{F}$ последовательности $\left(v_{t}^{l}\right)_{t=0}^{T},\left(v_{t}^{u}\right)_{t=0}^{T}$ по рекуррентным формулам

$$
\begin{aligned}
v_{T}^{l} & =v_{T}^{u}=\zeta_{T} \cdot \widehat{S}_{T}, \\
v_{t}^{u} & =-s\left(-\widehat{S}_{t} \mid G_{t}^{u}\right), \quad v_{t}^{l}=s\left(\widehat{S}_{t} \mid G_{t}^{l}\right), \\
G_{t}^{u} & =\left\{z \in \mathbf{R}^{d}: \mathbf{E}^{r}\left(\mathbf{I}\left(z \cdot \widehat{S}_{t+1} \geqslant v_{t+1}^{u}\right) \mid \mathscr{F}_{t}\right)(\omega, z)=1\right\}, \\
G_{t}^{l} & =\left\{z \in \mathbf{R}^{d}: \mathbf{E}^{r}\left(\mathbf{I}\left(z \cdot \widehat{S}_{t+1} \leqslant v_{t+1}^{l}\right) \mid \mathscr{F}_{t}\right)(\omega, z)=1\right\} .
\end{aligned}
$$

Eсли $G_{m}^{u}=\varnothing\left(\right.$ соответственно $\left.G_{m}^{l}=\varnothing\right)$ на множестве положительной меры для некоторого $m$, то $\mathbf{H}_{+}\left(\zeta_{T}\right)=\varnothing$ (соответственно $\left.\mathbf{H}_{-}\left(\zeta_{T}\right)=\varnothing\right)$. В противном случае

$$
\mathbf{H}_{+}\left(\zeta_{T}\right)=\left\{\zeta_{0} \in \mathbf{R}^{d}: \zeta_{0} \cdot \widehat{S}_{0} \geqslant v_{0}^{u}\right\}, \quad \mathbf{H}_{-}\left(\zeta_{T}\right)=\left\{\zeta_{0} \in \mathbf{R}^{d}: \zeta_{0} \cdot \widehat{S}_{0} \leqslant v_{0}^{l}\right\} .
$$

Д о к а з а т е л ь с т в о. В рассматриваемом случае $K_{t}^{*}=\left\{\lambda \widehat{S}_{t}: \lambda \geqslant\right.$ $0\}$. Проверим, что имеет место равенство

$$
v_{t}^{l}(\omega)=h_{t}^{l}\left(\omega, \widehat{S}_{t}(\omega)\right) \quad \text { п.н. }
$$

При $t=T$ это вытекает непосредственно из формул (1.5), (3.7). Пусть $v_{t+1}^{l}=h_{t+1}^{l}\left(\cdot, \widehat{S}_{t+1}\right)$ и $G_{t+1}^{l} \neq \varnothing$ п.н. В силу положительной однородности $h_{t+1}^{l}$ условие

$$
z \cdot \widehat{S}_{t+1} \leqslant v_{t+1}^{l}=h_{t+1}^{l}\left(\cdot, \widehat{S}_{t+1}\right)
$$


равносильно следующему: $z \cdot x^{*} \leqslant h_{t+1}^{l}\left(\cdot, x^{*}\right), x^{*} \in K_{t+1}^{*}$, и множество $G_{t}^{l}$, введенное в теореме 6 , совпадает с аналогичным множеством, определенным в теореме 2. Ясно, что отсюда вытекает равенство (3.9). Если $G_{t}^{l} \neq \varnothing$ п.н. для всех $t$, то по теореме 2 имеем

$$
\begin{aligned}
\mathbf{H}_{-} & =\left\{\zeta_{0}: \zeta_{0} \cdot x^{*} \leqslant h_{0}^{l}\left(x^{*}\right), x^{*} \in K_{0}^{*}\right\}=\left\{\zeta_{0}: \zeta_{0} \cdot \widehat{S}_{0} \leqslant h_{0}^{l}\left(\widehat{S}_{0}\right)\right\} \\
& =\left\{\zeta_{0}: \zeta_{0} \cdot \widehat{S}_{0} \leqslant v_{0}^{l}\right\} .
\end{aligned}
$$

Если $G_{m}^{l}=\varnothing$ на множестве положительной меры для некоторого $m$, то $\mathbf{H}_{-}=\varnothing$ по теореме 2 . Последовательность $\left(v_{t}^{u}\right)$ рассматривается аналогичным образом. Теорема доказана.

Как показано ниже, утверждения теорем 1 и 3 являются прямым следствием теорем 6 и 2 соответственно.

Д о к а з а т е л ь с в о т е о р е м ы 1 . Проведем доказательство для последовательности $\left(w_{t}^{u}\right)_{t=0}^{T}$. Рассмотрим модель валютного рынка без трения такую, что $S_{t}^{k}=\widehat{S}_{t}^{k} / \widehat{S}_{t}^{1}, k=2, \ldots, d$. Ограничиваясь рассмотрением платежного обязательства $\zeta_{T}=\left(f_{T}, 0, \ldots, 0\right)$ и переписывая рекуррентные соотношения теоремы 6 для дисконтированных величин $w_{t}^{u}=v_{t}^{u} / \widehat{S}_{t}^{1}$, находим, что последовательность $\left(w_{t}^{u}\right)_{t=0}^{T}$ удовлетворяет рекуррентным формулам теоремы 1. Остается проверить, что $w_{0}^{u}=\mathbf{C}^{*}\left(f_{T}\right)$.

В рассматриваемой модели ri $K_{t}^{*}=\left\{\lambda \widehat{S}_{t}: \lambda>0\right\}$ и для любого $\mathscr{F}_{t^{-}}$ измеримого вектора $Z_{t} \in \operatorname{ri} K_{t}^{*}$ имеет место представление

$$
Z_{t}=Z_{0}^{1} \rho_{t}\left(1, S_{t}^{2}, \ldots, S_{t}^{d}\right)
$$

где $\rho$ - строго положительный согласованный случайный процесс, который однозначно определяется процессом $Z$. Нетрудно проверить (см. [21]), что формула (3.10) устанавливает взаимно однозначное соответствие между процессами $Z / Z_{0}^{1}, Z \in \mathscr{M}\left(\right.$ ri $\left.K^{*}\right)$, и процессами плотностей $\rho_{t}=\mathbf{E}\left(d \mathbf{Q} / d \mathbf{P} \mid \mathscr{F}_{t}\right)$ эквивалентных мартингальных мер $\mathbf{Q}$ для $\left(S^{2}, \ldots, S^{d}\right): \mathbf{Q} \in \mathscr{P}(S)$. При этом формула (1.3) принимает вид

$$
\mathbf{H}_{+}=\left\{\zeta_{0} \in \mathbf{R}^{d}: \zeta_{0}^{1}+\sum_{i=2}^{d} \zeta_{0}^{i} S_{0}^{i} \geqslant \mathbf{E}_{\mathbf{Q}} f_{T}, \mathbf{Q} \in \mathscr{P}(S), \mathbf{E}_{\mathbf{Q}}\left|f_{T}\right|<\infty\right\}
$$

С другой стороны, первую формулу (3.8) можно привести к виду

$$
\mathbf{H}_{+}\left(\zeta_{T}\right)=\left\{\zeta_{0} \in \mathbf{R}^{d}: \zeta_{0}^{1}+\sum_{i=2}^{d} \zeta_{0}^{i} S_{0}^{i} \geqslant w_{0}^{u}\right\}
$$

Сопоставляя эту формулу с (3.11), заключаем, что имеет место равенство $w_{0}^{u}=\mathbf{C}^{*}\left(f_{T}\right)$, завершающие доказательство теоремы 1. 
Д о к аз а те ль с т в о т е о р е м ы 3 . В обозначениях теоремы 2 введем функции

$$
g_{t}^{l}\left(y^{*}\right)=h_{t}^{l}\left(1, y^{*}\right), \quad g_{t}^{u}\left(y^{*}\right)=h_{t}^{u}\left(1, y^{*}\right), \quad y^{*} \in C_{t} .
$$

Легко видеть, что данные функции удовлетворяют рекуррентным соотношениям теоремы 3 . Множества $G_{t}^{l}, G_{t}^{u}, \mathbf{H}_{+}, \mathbf{H}_{-}$, описанные в теоремax 2 и 3 , идентичны.

4. Примеры. Далее условие отсутствия арбитража (NA) (в примерах 4.1-4.3) и условие робастного отсутствия арбитража $\left(\mathrm{NA}^{r}\right)$ (в примерах 4.4, 4.5) предполагаются выполненными.

П р и м е р 4.1 (рынок без трения, конечное вероятностное пространство). Пусть $\Omega$ конечно и $\mathscr{F}_{t}$ порождается разбиением $\mathscr{D}_{t}$ с атомами $\left(D_{t}^{i}\right)_{i=1}^{m_{t}}$. Мера $\mathbf{P}$ предполагается невырожденной: $\mathbf{P}(D)>0$, $D \in \mathscr{D}_{T}$. Пусть атом $D_{t}^{i}$ разбиения $\mathscr{D}_{t}$ является объединением атомов $D_{t+1}^{j}, j \in \delta_{t}(i)$, разбиения $\mathscr{D}_{t+1}$. В работе [7] число элементов $\left|\delta_{t}(i)\right|$ множества $\delta_{t}(i)$ названо индексом ветвления атома $D_{t}^{i}$. Любое $\mathscr{F}_{t^{-}}$ измеримое отображение $F_{t}$ (однозначное или многозначное) постоянно на $D_{t}^{i}$. Положим для краткости $F_{t, i}=F_{t}(\omega), \omega \in D_{t}^{i}$.

Найдем множество $G_{t}^{u}$, указанное в теореме 1 . Элемент $(\alpha, \beta) \in$ $\mathbf{R} \times \mathbf{R}^{d-1}$ принадлежит $G_{t, i}^{u}$ тогда и только тогда, когда $\mathbf{P}\left\{\omega \in D_{t}^{i}: \alpha+\right.$ $\left.\beta \cdot S_{t+1} \geqslant w_{t+1}^{u}\right\}=\mathbf{P}\left(D_{t}^{i}\right)$. Другими словами,

$$
G_{t, i}^{u}=\left\{(\alpha, \beta) \in \mathbf{R} \times \mathbf{R}^{d-1}: \alpha+\beta \cdot S_{t+1, j} \geqslant w_{t+1, j}^{u}, j \in \delta_{t}(i)\right\}
$$

и $w_{t, i}^{u}$ совпадает с оптимальным значением целевой функции задачи линейного программирования

$$
\alpha+\left(\beta, S_{t, i}\right) \rightarrow \min _{\alpha, \beta}, \quad \alpha+\beta \cdot S_{t+1, j} \geqslant w_{t+1, j}^{u}, \quad j \in \delta_{t}(i),
$$

или двойственной задачи

$$
\begin{aligned}
& \sum_{j \in \delta_{t}(i)} w_{t+1, j}^{u} q_{j} \rightarrow \max _{q}, \\
& \sum_{j \in \delta_{t}(i)} S_{t+1, j} q_{j}=S_{t, i}, \quad \sum_{j \in \delta_{t}(i)} q_{j}=1, \quad q_{j} \geqslant 0, \quad j \in \delta_{t}(i) .
\end{aligned}
$$

Таким образом, задача вычисления верхней цены $\mathbf{C}^{*}$ платежного обязательства $f_{T}$ сводится к последовательности связанных задач, в каждой из которых требуется вычислить верхнюю цену платежного обязательства $w_{t+1}$ в одношаговой модели на редуцированном вероятностном пространстве, соответствующем атому разбиения $\mathscr{D}_{t}$. Данная процедура явно указана в работе [10]. В этой же работе содержатся задачи (4.1), (4.2). 
Рассмотрим более подробно случай двух активов $\left(d=2, S_{t}^{1}=1\right.$, $S_{t}^{2}=S_{t}$ ). Пусть на каждом атоме выполняется следующее условие невырожденности: $S_{t+1, j} \neq S_{t, i}, j \in \delta_{t}(i)$. Тогда максимум в (4.2) достигается на векторе $q_{t+1}^{*}$, имеющем в точности две ненулевые компоненты, поскольку таким свойством обладают все крайние точки многогранника, описывающего ограничения данной задачи. Введем относительнье иены $Y_{t}=S_{t} / S_{t-1}, t=1, \ldots, T$, и пусть

$$
I_{t}^{*}(i)=\left\{j \in \delta_{t}(i): q_{t+1, j}^{*} \neq 0\right\}
$$

обозначает двухэлементное множество «оптимальных» индексов. Имеем:

$$
\begin{gathered}
q_{t+1, j}=0, \quad j \in \delta_{t}(i) \backslash I_{t}^{*}(i), \\
q_{t+1, l}^{*}=\frac{1-Y_{t+1, k}}{Y_{t+1, l}-Y_{t+1, k}}, \quad q_{t+1, k}^{*}=\frac{Y_{t+1, l}-1}{Y_{t+1, l}-Y_{t+1, k}}, \quad k, l \in I_{t}^{*}(i), \\
w_{t, i}^{u}=q_{t+1, k}^{*} w_{t+1, k}^{u}+q_{t+1, l}^{*} w_{t+1, l}^{u} .
\end{gathered}
$$

Таким образом, верхняя цена хеджирования может быть вычислена с использованием специально подобранной биномиальной модели. Однако параметры такой модели зависят от платежного обязательства $f_{T}$. Явным образом результаты такого рода для многошаговой модели впервые были представлены, по-видимому, в работе [12]. Еще более конкретизируя задачу, предположим, что величины $Y_{t}$ независимы и $f_{T}=g_{T}\left(S_{T}\right), g_{T}(x):[0, \infty) \rightarrow \mathbf{R}$. Пусть $S_{t, i}$ строго возрастает по $i$. Покажем, что

$$
I_{t}^{*}(i)=\left\{\min \delta_{t}(i), \max \delta_{t}(i)\right\},
$$

если $g_{T}$ выпукла (как в случае опциона-колл) и

$$
I_{t}^{*}(i)=\left\{\max _{j}\left\{j \in \delta_{t}(i): Y_{t+1, j}<1\right\}, \min _{j}\left\{j \in \delta_{t}(i): Y_{t+1, j}>1\right\}\right\},
$$

если $g_{T}$ вогнута.

Предположим, что

$$
w_{t+1, i}^{u}=g_{t+1}\left(S_{t, i} Y_{t+1, i}\right), \quad i=1, \ldots, m_{t+1},
$$

где $g_{t+1}:[0, \infty) \rightarrow \mathbf{R}-$ выпуклая или вогнутая функция. Для каждого $i$ система уравнений

$$
\alpha+\beta S_{t, i} Y_{t+1, j}=g_{t+1}\left(S_{t, i} Y_{t+1, j}\right), \quad j \in I_{t}^{*}(i)
$$

имеет единственное решение $\left(\alpha^{*}, \beta^{*}\right)$, и это решение является допустимым для задачи (4.1):

$$
\alpha^{*}+\beta^{*} S_{t, i} Y_{t+1, j} \geqslant g_{t+1}\left(S_{t, i} Y_{t+1, j}\right), \quad j \notin I_{t}^{*}(i) .
$$


Действительно, если $g_{t+1}$ выпукла, то функция $g_{t+1}(y)-\beta^{*} y$ достигает максимума в одной из экстремальных точек $y=S_{t, i} Y_{t+1, j}$, что соответствует $j \in I_{t}^{*}(i)$.

В случае вогнутой функции $g_{t+1}$ определим $I_{t}^{*}(i)=\{k, l\}, l=k+1$, формулой (4.8). Пусть $j \geqslant l$. Проверим выполнение неравенства

$$
\begin{aligned}
& \alpha^{*}+\beta^{*} S_{t, i} Y_{t+1, j}-g_{t+1}\left(S_{t, i} Y_{t+1, j}\right) \\
& \quad=\beta^{*} S_{t, i}\left(Y_{t+1, j}-Y_{t+1, k}\right)+g_{t+1}\left(S_{t, i} Y_{t+1, k}\right)-g_{t+1}\left(S_{t, i} Y_{t+1, j}\right) \geqslant 0,
\end{aligned}
$$

равносильного неравенству (4.1). В силу вогнутости $g$ функция

$$
x \mapsto \frac{g(x)-g(a)}{x-a}, \quad x \neq a,
$$

является невозрастающей [11, теорема 1.3.1]. Следовательно,

$$
\frac{g_{t+1}\left(S_{t, i} Y_{t+1, j}\right)-g_{t+1}\left(S_{t, i} Y_{t+1, k}\right)}{S_{t, i}\left(Y_{t+1, j}-Y_{t+1, k}\right)} \leqslant \beta^{*}, \quad j \geqslant l,
$$

так как при $j=l$ имеет место равенство. Итак, неравенство в (4.1) при $j \geqslant l$ установлено. Случай $j \leqslant k$ рассматривается аналогично.

Таким образом, единственное решение $\left(\alpha_{t+1, i}^{*}, \beta_{t+1, i}^{*}\right)$ системы (4.10) является допустимым для задачи (4.1). С другой стороны, $q^{*}$, определяемое формулами (4.4), (4.5), является допустимым решением двойственной задачи $(4.2)$ и

$$
\left(\alpha_{t+1, i}^{*}+\beta_{t+1, i}^{*} S_{t+1, j}-w_{t+1, j}^{u}\right) q_{t+1, j}^{*}=0, \quad j \in \delta_{t}(i) .
$$

Суммируя данные равенства по $j \in \delta_{t}(i)$ (или используя вторую теорему двойственности $\left[26\right.$, гл. II, $\S 7$, п. 5]), заключаем, что $\left(\alpha_{t+1, i}^{*}, \beta_{t+1, i}^{*}\right)$, $\left(q_{t+1, j}^{*}\right)_{j \in \delta_{t}(i)}$ - оптимальные решения задач (4.1), (4.2) соответственно, так как значения целевых функций на этих решениях совпадают. Этим доказаны формулы (4.7), (4.8) в предположении (4.9).

Далее, в силу независимости $Y_{t+1}$ от $\mathscr{F}_{t}$, множества $(4.7),(4.8)$ не зависят от $i$ : $I_{t}^{*}(i)=I_{t}^{*}$. Это же касается оптимальных решений задач $(4.1),(4.2)$. Введем последовательность функций $\left(g_{t}\right)_{t=0}^{T}$ рекуррентной формулой

$$
g_{t}(x)=q_{t+1, k}^{*} g_{t+1}\left(x Y_{t+1, k}\right)+q_{t+1, l}^{*} g_{t+1}\left(x Y_{t+1, l}\right), \quad k, l \in I_{t}^{*} .
$$

Все функции $g_{t}$ выпуклы (соответственно вогнуты), если выпукла (соответственно вогнута) функция $g_{T}$. В предположении (4.9) в силу (4.6) имеем

$$
w_{t, i}=q_{t+1, k}^{*} g_{t+1}\left(S_{t, i} Y_{t+1, k}\right)+q_{t+1, l}^{*} g_{t+1}\left(S_{t, i} Y_{t+1, l}\right)=g_{t}\left(S_{t, i}\right)=g_{t}\left(S_{t-1, i} Y_{t, i}\right) .
$$


По индукции отсюда следует, что представление (4.9), а значит, и формулы (4.7), (4.8) верны для всех $t$.

Для наглядности наряду с исходной мультиномиальной моделью с независимыми относительными ценами $Y_{t}$ рассмотрим две «вложенные» биномиальные модели: 1) с относительными ценами $Y_{t}^{\prime}$, принимающими два экстремальных значения $Y_{t}$, и 2) с относительными ценами $Y_{t}^{\prime \prime}$, принимающими два значения $Y_{t}$, ближайших к 1 . Предыдущие рассуждения показывают, что верхняя цена хеджирования опциона $g_{T}\left(S_{T}\right)$ с выпуклой (соответственно вогнутой) платежной функцией $g_{T}$ в мультиномиальной модели равна цене того же опциона во «вложенной» биномиальной модели с относительными ценами $Y_{t}^{\prime}$ (соответственно $Y_{t}^{\prime \prime}$ ), ср. с [12].

П р и м е р 4.2 (рынок без трения, неограниченные относительные цены). Следуя [1], предположим, что условное распределение $\mu_{t}(\omega, d x)$ случайного вектора $S_{t+1}$ относительно $\mathscr{F}_{t}$ эквивалентно лебеговской мере на $\mathbf{R}_{+}^{d}$ для каждого $\omega$. Пусть $f_{T}=g_{T}\left(S_{T}\right)$, где $g_{T}: \mathbf{R}_{+}^{d-1} \rightarrow \mathbf{R}-$ непрерывная функция. В обозначениях теоремы 1 пара $(\alpha, \beta) \in \mathbf{R}^{d-1} \times \mathbf{R}$ принадлежит $G_{T-1}^{u}(\omega)$ тогда и только тогда, когда

$\mathbf{E}^{r}\left(\mathbf{I}\left(\alpha+\beta \cdot S_{T} \geqslant g_{T}\left(S_{T}\right)\right) \mid \mathscr{F}_{t}\right)=\mu_{T-1}\left(\omega,\left\{y: \alpha+\beta \cdot y \geqslant g_{T}(y)\right\}\right)=1 \quad$ п.н.

В силу непрерывности функции $g_{T}$ это равносильно условию

$$
\alpha+\beta \cdot y \geqslant g_{T}(y), \quad y \in \mathbf{R}_{+}^{d} .
$$

Следовательно, $w_{T-1}^{u}=g_{T-1}\left(S_{T-1}\right)$, где

$$
g_{T-1}(x)=\inf _{\alpha, \beta}\left\{\alpha+\beta \cdot x: \alpha+\beta \cdot y \geqslant g_{T}(y), y \in \mathbf{R}_{+}^{d}\right\} .
$$

Функция $g_{T-1}(x)$ вогнута. Более того, если $g_{T}$ вогнута, то $g_{T-1}=g_{T}$ (см. $\left[13\right.$, теорема 12.1]). По индукции отсюда следует, что $g_{0}=\cdots=g_{T-1}$ (при этом вогнутости $g_{T}$ не требуется) и

$$
\mathbf{C}^{*}\left(f_{T}\right)=w_{0}^{u}=g_{0}\left(S_{0}\right)=\inf _{\alpha, \beta}\left\{\alpha+\beta \cdot S_{0}: \alpha+\beta \cdot y \geqslant g_{T}(y), y \in \mathbf{R}_{+}^{d}\right\} .
$$

Аналогичным образом находим

$$
\mathbf{C}_{*}\left(f_{T}\right)=\sup _{\alpha, \beta}\left\{\alpha+\beta \cdot S_{0}: \alpha+\beta \cdot y \leqslant g_{T}(y), y \in \mathbf{R}_{+}^{d}\right\} .
$$

Как отмечено в работе [1], границы цен $f_{T}$ не зависят от числа шагов $T$. Для опциона-колл $f_{T}=\left(S_{T}-K\right)^{+}, d=2$, имеем

$$
\mathbf{C}_{*}\left(f_{T}\right)=\left(S_{0}-K\right)^{+}, \quad \mathbf{C}^{*}\left(f_{T}\right)=S_{0} .
$$


В случае $d=2$ для платежного обязательства $f_{T}=g_{T}\left(S_{T}\right)$ с непрерывной выпуклой функцией $g_{T}:[0,+\infty) \rightarrow \mathbf{R}$ справедливы формулы

$$
\begin{aligned}
& \mathbf{C}_{*}\left(f_{T}\right)=g_{T}\left(S_{0}\right), \\
& \mathbf{C}^{*}\left(f_{T}\right)=c_{0}+c_{1} S_{0}, \quad c_{0}=g_{T}(0), \quad c_{1}=\lim _{x \rightarrow+\infty} \frac{g_{T}(x)}{x} .
\end{aligned}
$$

Первая формула является следствием выпуклости $g_{T}$. Чтобы получить вторую, заметим, что если $\alpha, \beta$ удовлетворяют (4.13), то

$$
\alpha \geqslant c_{0}, \quad \beta \geqslant c_{1} .
$$

Обратно, если выполнены неравенства (4.16), то $\alpha, \beta$ удовлетворяют (4.13):

$$
\alpha+\beta x \geqslant c_{0}+c_{1} x \geqslant g_{T}(x) .
$$

Справедливость последнего неравенства вытекает из того, что функция

$$
y \mapsto \frac{g_{T}(y)-g_{T}(0)}{y}, \quad y>0,
$$

является неубывающей [11, теорема 1.3.1]. Минимизация линейной функции $\alpha+\beta S_{0}$ при ограничениях (4.16) дает формулу (4.15) для $\mathbf{C}^{*}\left(f_{T}\right)$.

В случае условно гауссовской модели для $\ln \left(S_{t+1} / S_{t}\right)$ формулы (4.14), (4.15) получены в работе [24], а в общем случае - в работе [6]. При произвольном $d$ верхняя цена хеджирования для ряда опционов, в том числе экзотических, вычислена в работе [1]. Тривиальность границ интервала безарбитражных цен в примере 4.2 связана с неограниченностью носителя $\mu_{t}$. В модели с ограниченными относительными ценами, которая рассматривается в следующем примере, границы цен могут быть вычислены по той же схеме, что и в примере 4.1.

П р и м е р 4.3 (один рисковый актив с ограниченныли относительными ченами). Пусть $d=2$ и $S_{t}=S_{0} \prod_{k=1}^{t} Y_{k}$. Обозначим через $\nu_{t}(\omega, d y)$ регулярное условное распределение $Y_{t+1}$ относительно $\mathscr{F}_{t}$. Предположим, что носитель $\operatorname{supp} \nu_{t}$ меры $\nu_{t}(\omega, \cdot)$ совпадает с интервалом $\left[a_{t}, b_{t}\right]$, где $0<a_{t}<1<b_{t}<\infty$, и $a_{t}, b_{t}$ не зависят от $\omega$. Это условие выполнено, в частности, если $Y_{t+1}$ не зависит от $\mathscr{F}_{t}$.

Рассмотрим платежное обязательство $f_{T}=g_{T}\left(S_{T}\right)$ с выпуклой функцией $g_{T}:[0, \infty) \rightarrow \mathbf{R}$. Предположим, что $w_{t+1}^{u}=g_{t+1}\left(S_{t+1}\right)$, где $g_{t+1}$ - выпуклая функция (при $t=T-1$ это выполняется по определению). Для почти всех $\omega$ верно, что пара $(\alpha, \beta) \in \mathbf{R} \times \mathbf{R}$ принадлежит $G_{t}^{u}(\omega)$ тогда и только тогда, когда

$\mathbf{E}^{r}\left(\mathbf{I}\left(\alpha+\beta S_{t} Y_{t+1} \geqslant g_{t+1}\left(S_{t} Y_{t+1}\right)\right) \mid \mathscr{F}_{t}\right)=\mu_{t}\left(\omega,\left\{\alpha+\beta S_{t} y \geqslant g_{t+1}\left(S_{t} y\right)\right\}\right)=1$. 
В силу непрерывности функции $g_{t+1}$ данное условие равносильно следующему:

$$
\alpha+\beta S_{t} y \geqslant g_{t+1}\left(S_{t} y\right), \quad y \in\left[a_{t}, b_{t}\right] .
$$

Но так как функция $g_{t+1}$ выпукла, неравенство (4.17) достаточно проверять только в граничных точках $a_{t}, b_{t}$. Таким образом, точная нижняя грань в выражении для $w_{t}^{u}$ (см. теорему 1 ) достигается на паре $\alpha_{t}, \beta_{t}$, являющейся решением системы уравнений

$$
\alpha+\beta S_{t} a_{t}=g_{t+1}\left(S_{t} a_{t}\right), \quad \alpha+\beta S_{t} b_{t}=g_{t+1}\left(S_{t} b_{t}\right),
$$

и

$$
w_{t}^{u}=\alpha_{t}+\beta_{t} S_{t}=g_{t}\left(S_{t}\right):=\frac{b_{t}-1}{b_{t}-a_{t}} g_{t+1}\left(S_{t} a_{t}\right)+\frac{1-a_{t}}{b_{t}-a_{t}} g_{t+1}\left(S_{t} b_{t}\right) .
$$

Определяемая этой формулой функция $g_{t}$ является выпуклой, и по индукции заключаем, что формула (4.18) верна для всех $t$.

Таким образом, верхняя цена хеджирования $\mathbf{C}^{*}\left(f_{T}\right)$ совпадает с ценой $f_{T}$ в соответствующей биномиальной модели с относительными ценами $Y_{t}^{\prime}$, принимающими значения $a_{t}, b_{t}$. Заметим, что для обоснования рекуррентного соотношения (4.18) достаточно выполнения условия $\left\{a_{t}, b_{t}\right\} \subset \operatorname{supp} \nu_{t} \subset\left[a_{t}, b_{t}\right]$.

Вычислим нижнюю цену хеджирования. Рассуждая как выше, находим

$$
w_{T-1}^{l}=\sup \left\{\alpha+\beta S_{T-1}: \alpha+\beta S_{T-1} y \leqslant g_{T}\left(S_{T-1} y\right), y \in\left[a_{T-1}, b_{T-1}\right]\right\} .
$$

В силу выпуклости $g_{T}$ отсюда вытекает, что $w_{T-1}^{l}=g_{T}\left(S_{T-1}\right)$ (см. $[13$, теорема 12.1]). По индукции,

$$
\mathbf{C}_{*}\left(f_{T}\right)=w_{0}^{l}=g_{T}\left(S_{0}\right) .
$$

Очевидное уточнение предыдущих рассуждений показывает, что для справедливости формулы $w_{T-1}^{l}=g_{T}\left(S_{T-1}\right)$, а значит, и $(4.19)$, достаточно выполнения условия $1 \in \operatorname{supp} \nu_{t}$ вместо $\operatorname{supp} \nu_{t}=\left[a_{t}, b_{t}\right]$.

Результаты, аналогичные представленным в примере 4.3 , получены в [4], [9], [25][гл. V, § 1с], [6], [20].

П р и м е р 4.4 (модель рынка с трением при наличии банковского счета, конечное вероятностное пространство). В обозначениях примера 4.1 формулы теоремы 3 для верхней цены хеджирования принимают вид

$$
\begin{aligned}
g_{T, i}^{u}\left(y^{*}\right) & =\xi_{T, i}+\eta_{T, i} \cdot y^{*}, \quad y^{*} \in C_{T, i}, \\
g_{t, i}^{u}\left(y^{*}\right) & =\inf _{\alpha, \beta}\left\{\alpha+\beta \cdot y^{*}: \alpha+\beta \cdot z^{*} \geqslant g_{t+1, j}^{u}\left(z^{*}\right),\right. \\
\left.z^{*} \in C_{t+1, j}, j \in \delta_{t}(i)\right\}, \quad y^{*} \in C_{t, i}, & \\
\mathbf{C}^{*}\left(\xi_{T}, \zeta_{T}\right) & =\sup \left\{g_{0}^{u}\left(y^{*}\right): y^{*} \in C_{0}\right\} .
\end{aligned}
$$


Нетрудно проверить, что при $d=2$ они совпадают с формулами работы [19] и, как указано в [19], обобщают известные алгоритмы вычисления верхней цены хеджирования.

Для иллюстрации рассмотрим биномиальную модель $\left(\left|\delta_{t}(i)\right|=2\right)$ с «малыми» операционными издержками $\left(S_{t, k}^{a} \leqslant S_{t, l}^{b}, k<l\right)$. Из указанных рекуррентных формул следует, что если функции $g_{t+1, j}^{u}$ линейны на $\left[S_{t+1, j}^{b}, S_{t+1, j}^{a}\right], j \in \delta_{t}(i)$, то и функция $g_{t, i}^{u}$ линейна на $\left[S_{t, i}^{b}, S_{t, i}^{a}\right]$, поскольку интервалы

$$
\left(S_{t+1, j}^{b}, S_{t+1, j}^{a}\right), \quad j \in \delta_{t}(i),
$$

не пересекаются. Пусть $k, l \in \delta_{t}(i)$. Чтобы найти значения $g_{t, i}^{u}\left(S_{t, i}^{r}\right)$, $r \in\{a, b\}$, полностью определяющие функцию $g_{t, i}^{u}$, рассмотрим четыре прямые, проходящие через одну из точек

$$
A_{t+1, k}^{r}=\left(S_{t+1, k}^{r}, g_{t+1, k}^{u}\left(S_{t+1, k}^{r}\right)\right), \quad r \in\{a, b\},
$$

и одну из точек $B_{t+1, l}^{r}=\left(S_{t+1, l}^{r}, g_{t+1, l}^{u}\left(S_{t+1, l}^{r}\right)\right), r \in\{a, b\}$. Среди точек указанных прямых с абсциссами $S_{t, i}^{r}, r \in\{a, b\}$, выберем ту, которая имеет наибольшую ординату $y_{t, i}^{r}, r \in\{a, b\}$. Ясно, что значение $g_{t, i}\left(S_{t, i}^{r}\right)$, $r \in\{a, b\}$, совпадает с $y_{t, i}^{r}$ :

$$
\begin{aligned}
g_{t, i}^{u}\left(S_{t, i}^{r}\right)=\max _{p, q \in\{a, b\}}( & \frac{S_{t+1, l}^{q}-S_{t, i}^{r}}{S_{t+1, l}^{q}-S_{t+1, k}^{p}} g_{t+1, k}^{u}\left(S_{t+1, k}^{p}\right) \\
& \left.+\frac{S_{t, i}^{r}-S_{t+1, k}^{p}}{S_{t+1, l}^{q}-S_{t+1, k}^{p}} g_{t+1, l}^{u}\left(S_{t+1, l}^{q}\right)\right)
\end{aligned}
$$

при $r \in\{a, b\}, k, l \in \delta_{t}(i)$. По индукции эта формула верна для всех $t$. Заметим, что равенство (4.21) вытекает также из рассмотрения задачи линейного программирования, двойственной к той, которая определяет функцию $g_{t, i}^{u}$.

Формула (4.20) принимает вид

$$
\mathbf{C}^{*}\left(\xi_{T}, \zeta_{T}\right)=\max \left\{g_{0}^{u}\left(S_{0}^{b}\right), g_{0}^{u}\left(S_{0}^{a}\right)\right\} .
$$

Формулы (4.21), (4.22) совпадают с полученными в работе [23].

П р и м е р 5.5 (опцион-колл в модели с неограниченными относительными ченами при наличии трения). Пусть в модели с банковским счетом $d=2, S_{t}^{b}=\left(1-b_{t}\right) S_{t}, S_{t}^{a}=\left(1+a_{t}\right) S_{t}$, где $a_{t} \geqslant 0, b_{t} \in[0,1)$ неслучайны. Обозначим через $\mu_{t}(\omega, d z)$ регулярное условное распределение $S_{t+1}$ относительно $\mathscr{F}_{t}=\sigma\left(S_{0}, \ldots, S_{t}\right)$ и предположим, что мера $\mu_{t}(\omega, d z)$ эквивалентна мере Лебега на $\mathbf{R}_{+}$. Воспользовавшись теоремой 3 , при этих предположениях вычислим верхнюю и нижнюю цены хеджирования опциона-колл с поставкой:

$$
\xi_{T}=-K \mathbf{I}\left\{S_{T}^{b} \geqslant K\right\}, \quad \eta_{T}=\mathbf{I}\left\{S_{T}^{b} \geqslant K\right\} .
$$


При сделанных предположениях функции $g_{t}^{l}, g_{t}^{u}$, указанные в теореме 3 , имеют вид $g_{t}^{l}=g_{t}^{l}\left(S_{t}, y^{*}\right), g_{t}^{u}=g_{t}^{u}\left(S_{t}, y^{*}\right)$. Положим

$$
\begin{aligned}
& \chi_{t}^{u}\left(S_{t}, \beta\right)=\sup \left\{g_{t}^{u}\left(S_{t}, y^{*}\right)-\beta y^{*}: y^{*} \in\left[\left(1-b_{t}\right) S_{t},\left(1+a_{t}\right) S_{t}\right]\right\}, \\
& \chi_{t}^{l}\left(S_{t}, \beta\right)=\inf \left\{g_{t}^{l}\left(S_{t}, y^{*}\right)-\beta y^{*}: y^{*} \in\left[\left(1-b_{t}\right) S_{t},\left(1+a_{t}\right) S_{t}\right]\right\} .
\end{aligned}
$$

Тогда

$$
\begin{aligned}
G_{t}^{u} & =\left\{(\alpha, \beta): \mathbf{E}^{r}\left(\mathbf{I}\left\{\alpha \geqslant \chi_{t+1}^{u}\left(S_{t+1}, \beta\right)\right\} \mid \mathscr{F}_{t}\right)=1\right\}, \\
G_{t}^{l} & =\left\{(\alpha, \beta): \mathbf{E}^{r}\left(\mathbf{I}\left\{\alpha \leqslant \chi_{t+1}^{l}\left(S_{t+1}, \beta\right)\right\} \mid \mathscr{F}_{t}\right)=1\right\} .
\end{aligned}
$$

Вычислим верхнюю цену хеджирования. Поскольку $g_{T}^{u}\left(S_{T}, y^{*}\right)=$ $\xi_{T}+\eta_{T} y^{*}, y^{*} \in\left[S_{T}^{b}, S_{T}^{a}\right]$, то

$$
\chi_{T}^{u}\left(S_{T}, \beta\right)=\xi_{T}+S_{T}^{a}\left(\eta_{T}-\beta\right)^{+}-S_{T}^{b}\left(\eta_{T}-\beta\right)^{-} .
$$

Следовательно, $(\alpha, \beta) \in G_{T-1}^{u}$ тогда и только тогда, когда

$$
\mathbf{E}^{r}\left(\mathbf{I}\left\{\alpha \geqslant \chi_{T}^{u}\left(S_{T}, \beta\right)\right\} \mid \mathscr{F}_{T-1}\right)=\mu_{T-1}\left(\omega,\left\{z: \alpha \geqslant \chi_{T}^{u}(z, \beta)\right\}\right)=1 .
$$

Отсюда следует, что точки $(\alpha, \beta) \in G_{T-1}^{u}$ описываются неравенством

$$
\begin{aligned}
\alpha \geqslant \chi_{T}^{u}(z, \beta)= & -K \mathbf{I}\left\{\left(1-b_{T}\right) z \geqslant K\right\} \\
& +\left(\mathbf{I}\left\{\left(1-b_{T}\right) z \geqslant K\right\}-\beta\right)^{+}\left(1+a_{T}\right) z \\
& -\left(\mathbf{I}\left\{\left(1-b_{T}\right) z \geqslant K\right\}-\beta\right)^{-}\left(1-b_{T}\right) z, \quad z \geqslant 0 .
\end{aligned}
$$

При $\beta<1$ правая часть данного неравенства неограничена сверху, а при $\beta \geqslant 1$ она неположительна и обращается в нуль при $z=0$. Таким образом, $G_{T-1}^{u}=[0, \infty) \times[1, \infty)$ и

$$
g_{T-1}^{u}\left(y^{*}\right)=\inf \left\{\alpha+\beta y^{*}:(\alpha, \beta) \in G_{T-1}^{u}\right\}=y^{*}, \quad y^{*} \in\left[S_{T-1}^{b}, S_{T-1}^{a}\right] .
$$

Далее,

$\chi_{T-1}^{u}\left(S_{T-1}, \beta\right)=\sup \left\{y^{*}-\beta y^{*}: y^{*} \in\left[S_{T-1}^{b}, S_{T-1}^{a}\right]\right\}=(1-\beta)^{+} S_{T-1}^{a}-(1-\beta)^{-} S_{T}^{b}$

и $(\alpha, \beta) \in G_{T-1}^{u}$ тогда и только тогда, когда

$$
\alpha \geqslant(1-\beta)^{+}\left(1+a_{T-1}\right) z-(1-\beta)^{-}\left(1-b_{T-1}\right) z, \quad z \geqslant 0,
$$

что равносильно условиям $\alpha \geqslant 0, \beta \geqslant 1$. Следовательно,

$$
G_{T-2}^{u}=G_{T-1}^{u}, \quad g_{T-2}^{u}=y^{*}, \quad y^{*} \in\left[S_{T-2}^{b}, S_{T-2}^{a}\right] .
$$


По индукции, $g_{0}\left(y^{*}\right)=y^{*}, y^{*} \in\left[S_{0}^{b}, S_{0}^{a}\right]$, и по формуле (1.7)

$$
\mathbf{C}^{*}\left(\xi_{T}, \eta_{T}\right)=S_{0}^{a} \text {. }
$$

Перейдем к вычислению нижней цены хеджирования. Имеем:

$$
\begin{aligned}
\chi_{T}^{l}\left(S_{T}, \beta\right) & =\xi_{T}+\inf \left\{\left(\eta_{T}-\beta\right) y^{*}: y^{*} \in\left[S_{T}^{b}, S_{T}^{a}\right]\right\} \\
& =\xi_{T}+S_{T}^{b}\left(\eta_{T}-\beta\right)^{+}-S_{T}^{a}\left(\eta_{T}-\beta\right)^{-} .
\end{aligned}
$$

Множество $G_{T-1}^{l}$ состоит из точек $(\alpha, \beta)$, удовлетворяющих условию $\alpha \leqslant$ $\chi_{T}^{l}(z, \beta), z \geqslant 0$, где

$$
\begin{aligned}
\chi_{T}^{l}(z, \beta)= & -K \mathbf{I}\left\{\left(1-b_{T}\right) z \geqslant K\right\}+\left(\mathbf{I}\left\{\left(1-b_{T}\right) z \geqslant K\right\}-\beta\right)^{+}\left(1-b_{T}\right) z \\
& -\left(\mathbf{I}\left\{\left(1-b_{T}\right) z \geqslant K\right\}-\beta\right)^{-}\left(1+a_{T}\right) z .
\end{aligned}
$$

Простое вычисление показывает, что

$$
\inf _{z>0} \chi_{T}^{l}(z, \beta)= \begin{cases}-\infty, & \beta>1 ; \\ -K \frac{1+a_{T}}{1-b_{T}} \beta, & \beta \in[0,1] ; . \\ 0, & \beta<0 .\end{cases}
$$

Следовательно,

$$
\begin{aligned}
G_{T-1}^{l} & =\{\alpha \leqslant 0, \beta<0\} \cap\left\{\alpha \leqslant-K \frac{1+a_{T}}{1-b_{T}} \beta, \beta \in[0,1]\right\} \\
g_{T-1}^{l} & =\sup _{\alpha, \beta}\left\{\alpha+\beta y^{*}:(\alpha, \beta) \in G_{T-1}^{l}\right\}=\max _{\beta \in[0,1]}\left\{\beta\left(y^{*}-K \frac{1+a_{T}}{1-b_{T}}\right)\right\} \\
& =\left(y^{*}-K^{\prime}\right)^{+}, \quad y^{*} \in\left[S_{T-1}^{b}, S_{T-1}^{a}\right], \quad K^{\prime}=K \frac{1+a_{T}}{1-b_{T}} .
\end{aligned}
$$

Далее,

$$
\begin{aligned}
\chi_{T-1} & \left(S_{T-1}, \beta\right)=\inf \left\{g_{T-1}^{l}\left(y^{*}\right)-\beta y^{*}: y^{*} \in\left[S_{T-1}^{b}, S_{T-1}^{a}\right]\right\} \\
= & -\beta S_{T-1}^{a} \mathbf{I}\left(K^{\prime}>S_{T-1}^{a}\right) \\
& +\min \left\{-\beta K^{\prime},-K^{\prime}+(1-\beta)^{+} K^{\prime}-(1-\beta)^{-} S_{T-1}^{a}\right\} \mathbf{I}\left(S_{T-1}^{b} \leqslant K^{\prime} \leqslant S_{T-1}^{a}\right) \\
& +\left(-K^{\prime}+(1-\beta)^{+} S_{T-1}^{b}-(1-\beta)^{-} S_{T-1}^{a}\right) \mathbf{I}\left(K^{\prime}<S_{T-1}^{b}\right) .
\end{aligned}
$$

Анализируя соответствующее выражение для $\chi_{T-1}^{l}(z, \beta)$, находим

$$
\inf _{z>0} \chi_{T-1}^{l}(z, \beta)=\inf _{z>0} \chi_{T}^{l}(z, \beta) .
$$

Следовательно, $G_{T-2}^{l}=G_{T-1}^{l}$ и $g_{T-2}^{l}=\left(y^{*}-K^{\prime}\right)^{+}$при $y^{*} \in\left[S_{T-2}^{b}, S_{T-2}^{a}\right]$. По индукции, $g_{0}^{l}\left(y^{*}\right)=\left(y^{*}-K^{\prime}\right)^{+}$при $y^{*} \in\left[S_{0}^{b}, S_{0}^{a}\right]$ и

$$
\mathbf{C}_{*}\left(\xi_{T}, \eta_{T}\right)=\left(S_{0}^{b}-K \frac{1+a_{T}}{1-b_{T}}\right)^{+}
$$

по формуле (1.8). 
Формулы (4.23), (4.24) обобщают формулы (4.14) примера 4.2, полученные в предположении отсутствия трения. Отметим, что найденные границы цен являются тривиальными и соответствуют простым стратегиям хеджирования. Продавец опциона-колл получает $S_{0}^{a}$ единиц банковского счета и переводит эти деньги в рисковый актив. Если $S_{T}^{b} \geqslant K$, то он передает рисковый актив владельцу опциона.

Покупатель опциона-колл становится обладателем долга в $\left(S_{0}^{b}-\right.$ $\left.K^{\prime}\right)^{+}$единиц банковского счета. Интересен лишь случай $S_{0}^{b}>K^{\prime}$. Осуществляя короткую продажу единицы рискового актива, инвестор получает $S_{0}^{b}$ единиц банковского счета. В момент времени $T$ его банковский счет будет составлять

$$
S_{0}^{b}-\left(S_{0}^{b}-K^{\prime}\right)^{+}-K \mathbf{I}\left(S_{T}^{b} \geqslant K\right)=K\left(\frac{1+a_{T}}{1-b_{T}}-\mathbf{I}\left(S_{T}^{b} \geqslant K\right)\right) \geqslant 0,
$$

а количество единиц рискового актива будет равно $-1+\mathbf{I}\left(S_{T}^{b} \geqslant K\right)=$ $-\mathbf{I}\left(S_{T}^{b}<K\right)$. Количество единиц банковского счета, необходимых для покрытия рисковой части портфеля, составляет

$$
S_{T}^{a} \mathbf{I}\left(S_{T}^{b}<K\right)=S_{T}^{b} \frac{1+a_{T}}{1-b_{T}} \mathbf{I}\left(S_{T}^{b}<K\right)
$$

и не превосходит (4.25).

Тривиальность верхней цены хеджирования в модели Блэка-Шоулза с операционными издержками установлена в [22].

\section{СПИСОК ЛИТЕРАТУРЫ}

1. Carassus L., Gobet E., Temam E. A class of financial products and models where super-replication prices are explicit. - Proceedings of the 6th Ritsumeikan international symposium on stochastic processes and applications to mathematical finance (Kyoto, Japan, 2006). Hackensack, World Scientific, 2007, p. 67-84.

2. Delbaen F., Schachermayer W. The Mathematics of Arbitrage. Berlin: Springer, 2006, $373 \mathrm{p}$.

3. Дынкин Е. Б., Евстигнеев И. В. Регулярные условные математические ожидания соответствий. - Теория вероятн. и ее примен., 1976, т. 21, в. 2, с. 334-347.

4. Гапеев П. В. Расчет верхних и нижних цен опционов Европейского типа. Успехи матем. наук, 1997, т. 52, № 4, с. 199-200.

5. Grigoriev P. G. On low dimensional case in the fundamental asset pricing theorem with transaction costs. - Statist. Decisions, 2005, v. 23, № 1, p. 33-48.

6. Гущин А. А., Мордецкий Э. Границы цен опционов для семимартингальных моделей рынка. - Тр. МИАН, 2002, т. 237, с. 80-122.

7. Harrison J.M., Pliska S.R. Martingales and stochastic integrals in the theory of continuous trading. - Stochastic Process. Appl., 1981, v. 11, № 3, p. 215-260.

8. Kabanov Yu., Safarian M. Markets with Transaction Costs. Berlin: Springer-Verlag, 2009, $294 \mathrm{p}$

9. Kascheev D. E. On the option pricing for a generalization of the binomial model. J. Math. Sci. (New York), 2000, v. 99, № 3, p. 1267-1272. 
10. Мельников А. В., Феоктистов К. М. Вопросы безарбитражности и полноты дискретных рынков и расчеты платежных обязательств. - Обозр. прикл. и промышл. матем., 2001, т. 8, в. 1, с. 28-40.

11. Niculescu C. P., Persson L.-E. Convex Functions and Their Applications. A Contemporary Approach. New York: Springer, 2006, 255 p.

12. Ritchken P. H., Kuo S. Option bounds with finite revision opportunities. - J. Finance, 1988, v. 43, № 2, p. 301-308.

13. Рокафеллар Р. Т. Выпуклый анализ. М.: Мир, 1973, 469 с.

14. Rockafellar R. T., Wets R.J.-B. Variational Analysis. Berlin: Springer, 1998, 733 p.

15. Рохлин Д. Б. Задача о мартингальном выборе в случае конечного дискретного времени. - Теория вероятн. и ее примен., 2005, т. 50, в. 3, с. 480-500.

16. Рохлин Д. Б. Конструктивный критерий отсутствия арбитража при наличии операционных издержек в случае конечного дискретного времени. - Теория вероятн. и ее примен., 2007, т. 52 , в. 1, с. $41-59$.

17. Рохлин Д.Б. Теорема о мартингальном выборе для случайной последовательности с относительно открытыми выпуклыми значениями. - Матем. заметки, 2007, т. 81 , в. 4 , с. $614-620$.

18. Rokhlin D. B. Martingale selection problem and asset pricing in finite discrete time. Electron. Comm. Probab., 2007, v. 12, p. 1-8.

19. Roux A., Tokarz K., Zastawniak T. Options under proportional transaction costs: An algorithmic approach to pricing and hedging. - Acta Appl. Math., 2008, v. 103, № 2, p. 201-219.

20. Rüschendorf L. On upper and lower prices in discrete time-models. - Тр. МИАН, 2002, v. 237, p. 134-139.

21. Schachermayer $W$. The fundamental theorem of asset pricing under proportional transaction costs in finite discrete time. - Math. Finance, 2004, v. 14, № 1, p. 19-48.

22. Soner H.M., Shreve S.E., Cvitanić J. There is no nontrivial hedging portfolio for option pricing with transaction costs. - Ann. Appl. Probab., 1995, v. 5, № 2, p. 327355.

23. Tokarz K., Zastawniak T. Dynamic programming algorithms for the ask and bid prices of American options under small proportional transaction costs. - Working paper, 2004, available at SSRN: http://ssrn.com/abstract $=581543$.

24. Шатаев О. В. О справедливой цене опциона европейского типа. - Успехи матем. наук, 1998 , т. 53, № 6, с. 269-270.

25. Ширяев A.Н. Основы стохастической финансовой математики. Т. 2: Теория. М.: Фазис, 1998, 528 с.

26. Зуховичкий С.И., Авдеева Л. И. Линейное и выпуклое программирование. М.: Наука, 1967, 460 c. 